\title{
ABSOLUTE CONVERGENCE OF DOUBLE TRIGONOMETRIC FOURIER SERIES AND WALSH-FOURIER SERIES
}

\author{
PhD THESIS
}

ANTAL VERES

SUPERVISOR:

FERENC MÓRICZ DSc

UNIVERSITY OF SZEGED

FACULTY OF SCIENCE AND INFORMATICS

BOLYAI INSTITUTE

DOCTORAL SCHOOL

IN MATHEMATICS AND COMPUTER SCIENCE

SZEGED 


\section{Contents}

$\begin{array}{ll}\text { Introduction } & 1\end{array}$

1 Trigonometric Fourier series $\quad 3$

1.1. Known results on single Fourier series . . . . . . . . . . . . . . 3

1.2. New results on double Fourier series . . . . . . . . . . . . . 8

1.3. Absolute convergence of double Fourier series . . . . . . . . 24

1.4. Extension to multiple Fourier series . . . . . . . . . . . 27

2 Walsh-Fourier series $\quad 31$

2.1. Background: the Walsh-Paley system . . . . . . . . . . . . 31

2.2. Known results on single Walsh-Fourier series . . . . . . . . 32

2.3. New results on double Walsh-Fourier series . . . . . . . . . . 36

2.4. Absolute convergence of double Walsh-Fourier series . . . . . . 46

$\begin{array}{ll}\text { Summary } & 50\end{array}$ 


\section{Acknowledgements}

The author wishes to express his gratitude to Professor Ferenc Móricz for many insightful conversation, constant support and encouragement. 


\section{Introduction}

Fourier series were introduced by Joseph Fourier (1768 - 1830) for the purpose of solving the heat equation in a metal plate. The heat equation is a partial differential equation. Prior to Fourier's work, no solution to the heat equation was known in the general case, although particular solutions were known if the heat source behaved in a simple way, in particular, if the heat source was a sine or cosine wave. These simple soulutions are sometimes called eigensolutions. Fourier's idea was to model a complicated heat source as a superposition of simple sine and cosine waves, and to write the solution as a superposition of the correponding eigensolutions. This superposition or linear combination is called the Fourier series.

Although the original motivation was to solve the heat equation, it later became obvious that the same techniques could be applied to a wide array of mathematical and physical problems, for example in electrical engineering, vibration analysis, acoustic, optics, signal and image processing, quantum mechanics, econometrics, etc.

The developement of the theory of Fourier series in mathematical analysis began in the 19th century and it has been a source of new ideas for analysis during the last two centuries, and is likely to be so in years to come. Many basic notions and results in the theory of functions have been obtained using Fourier or more generally trigonometric series. For example, the generally accepted notion of function was first formulated by Dirichlet dealing with the convergence of Fourier series, or the the definition of the Riemann integral in its general form appeared in Riemann's "Habilitatinschrift" devoted to 
trigonometric series. In more recent times many central notions of mathematics were developed in close connection to Fourier series, just to mention the theory of sets, the Lebesgue intergal and the theory of generalized funtions (distributions).

Pointweise and uniform convergence are central problems in Fourier theory. In the last century several results were published in connection of convergence of Fourier series, among others the theorems of Bernstein and Zygmund relatives to absolute convergence. These theorems gave sufficient conditions for the absolute and so for the uniform convergence of the Fourier series of a complex valued periodic function with period $2 \pi$ in terms of moduli of continuity, of bounded variation and in he case when the function is absolutely continuous. In [4] Gogoladze and Meskhia proved these theorems in a more general framework. In the first part of our thesis we extend these results from single to multiple Fourier series.

The Walsh functions form a complete orthonormal system whic can be applied in many situation, The Walsh system can perform all the usual applications of trigonometric system mentioned above and can perform them more efficiently. Also, they are easy to implemet on computers and can be used with very little storeage space. This is due in part to the fact that the Walsh functions take on only the values +1 and -1 . The Walsh system is also interesting from a theoretical point of view, as well. For more details, see for example [10].

In the second part of our thesis we give sufficient conditions for the absolute convergence of double Walsh-Fourier series. More generally, we extend the results of F. Móricz [5] from single to double Walsh-Fourier series. 


\section{Chapter 1}

\section{Trigonometric Fourier series}

\subsection{Known results on single Fourier series}

Let $f=f(x)$ be a complex-valued periodic function with period $2 \pi$. We recall that if $f \in \mathrm{L}^{1}(\mathbb{T})$, where $\mathbb{T}:=[-\pi, \pi)$ is the one-dimensional torus, then the Fourier series of $f$ is given by

$$
f(x) \sim \sum_{m \in \mathbb{Z}} \hat{f}(m) e^{i m x}, \quad x \in \mathbb{T},
$$

where the Fourier coefficients $\hat{f}(m)$ are defined by

$$
\hat{f}(m):=\frac{1}{2 \pi} \int_{\mathbb{T}} f(x) e^{-i m x} d x, \quad m \in \mathbb{Z} .
$$

We recall that the integral modulus of continuity (in the norm of $\mathrm{L}^{p}$ ) of a function $f \in \mathrm{L}^{p}(\mathbb{T})$ for some $1 \leq p<\infty$ is defined by

$$
\omega(f ; \delta)_{p}:=\sup \left\{\left(\frac{1}{2 \pi} \int_{\mathbb{T}}|f(x+h)-f(x)|^{p} d x\right)^{1 / p}: 0<h \leq \delta\right\} ;
$$

while the ordinary modulus of continuity of a function $f \in \mathrm{C}(\mathbb{T})$ is defined by

$$
\omega(f ; \delta):=\sup \{|f(x+h)-f(x)|: x \in \mathbb{T}, 0<h \leq \delta\} .
$$

Following the definition in [4], a sequence $\gamma=\left\{\gamma_{m}: m \in \mathbb{N}_{+}\right\}$of nonnegative numbers is said to belong to the class $\mathfrak{A}_{a}$ for some $a \geq 1$ if 


$$
\left(\sum_{m \in D_{\mu}} \gamma_{m}^{\alpha}\right) 1 / \alpha \leq \kappa 2^{\mu(1-\alpha) / \alpha} \sum_{m \in D_{\mu-1}} \gamma_{m}, \quad \mu \in \mathbb{N}_{+}
$$

where

$$
D_{0}:=\{1\}, \quad D_{\mu}:=\left\{2^{\mu-1}+1,2^{\mu-1}+2, \ldots, 2^{\mu}\right\}, \quad \mu \in \mathbb{N}_{+},
$$

and the constans $\kappa$ does not depend on $\mu$. Without loss of generality, we may assume that $\kappa \geq 1$.

It is easy to check that

$$
\mathfrak{A}_{\alpha_{2}} \subset \mathfrak{A}_{\alpha_{1}} \quad \text { whenever } \quad 1 \leq \alpha_{1}<\alpha_{2}<\infty \text {. }
$$

If a sequence $\gamma=\left\{\gamma_{m} \geq 0\right\}$ is such that the inequality

$$
\max \left\{\gamma_{m}: m \in D_{\mu}\right\} \leq \kappa \min \left\{\gamma_{m}: m \in D_{\mu-1}\right\}, \quad \mu \in \mathbb{N}_{+},
$$

holds, where $\kappa$ is a constant, then $\gamma \in \mathfrak{A}_{\alpha}$ for every $\alpha \geq 1$. Such an inequality was first considered by Ul'yanov [13]. Furthermore, if $\gamma=\left\{\gamma_{m}\right\}$ is of the form

$$
\gamma_{m}=m^{\beta} w(m), \quad m \in \mathbb{N}_{+}
$$

where $\beta \in \mathbb{R}$ and $w: \mathbb{R}_{+} \rightarrow \mathbb{R}_{+}$is a slowly varying function, that is

$$
\lim _{x \rightarrow \infty} \frac{w(\lambda x)}{w(x)}=1 \text { for every } 0<\lambda<\infty
$$

then $\gamma \in \mathcal{A}_{\alpha}$ for every $\alpha \geq 1$.

We summarize the latest results related to the absolute convergence of single Fourier series obtained in [4] by Gogoladze and Meskhia. We agree to put

$$
\gamma_{-m}:=\gamma_{m}, \quad m \in \mathbb{N}_{+}
$$


Theorem 1.1. Suppose $f \in L^{p}(\mathbb{T})$ for some $1<p \leq 2$. If

$$
\gamma=\{\gamma\} \in \mathfrak{A}_{p /(p-r p+r)} \quad \text { for some } \quad r \in(0, q)
$$

where $1 / p+1 / q=1$, then

$$
\sum(\gamma ; f)_{r}:=\sum_{|m| \geq 1} \gamma_{m}|\hat{f}(m)|^{r} \leq \kappa C \sum_{\mu=0}^{\infty} 2^{-\mu r / q} \Gamma_{\mu-1} \omega^{r}\left(f ; \frac{\pi}{2^{\mu}}\right)_{p}
$$

where $\kappa$ is from (1.1) corresponding to $\alpha:=p /(p-r p+r), C$ is a constant,

$$
\Gamma_{\mu}:=\sum_{m \in D_{\mu}} \gamma_{m} \quad \text { for } \quad \mu \in \mathbb{N}, \quad \text { and } \quad \Gamma_{-1}:=\Gamma_{0}=\left\{\gamma_{1}\right\}
$$

Corollary 1.1. Under the conditions of Theorem 1.1, we have

$$
\sum(\gamma ; f)_{r} \leq \kappa C \sum_{m=1}^{\infty} m^{-r / q} \gamma_{m} \omega^{r}\left(f ; \frac{\pi}{m}\right)_{p} .
$$

We denote by $C$ a constant whose value may be different at each occurrence.

In the case when $\gamma_{m} \equiv 1$ and $r=1$, it follows from Corollary 1 that

$$
\sum(1 ; f)_{1}:=\sum_{|m| \geq 1}|\hat{f}(m)| \leq \kappa C \sum_{m=1}^{\infty} m^{-1 / q} \omega\left(f ; \frac{\pi}{m}\right)_{p} .
$$

In particular, if $f \in L^{p}(\mathbb{T})$ for some $1<p \leq 2$ and

$$
\omega\left(f ; \frac{\pi}{m}\right)_{p}=O\left(m^{-\alpha}\right) \quad \text { for some } \quad \alpha>\frac{1}{p}
$$

then the Fourier series of $f$ is absolutely convergent.

Let $\gamma_{m} \equiv 1$. Corollary 1.1 was proved by Bernstein [2] in the case when $f \in \operatorname{Lip}(\alpha)$ for some $\alpha>1 / 2$ and $r=1$; by Szász [12] in the case when 
$p=2$ and $r>2 /(2 \alpha+1) ;$ and also by Szász [12] in the case when $1<p \leq 2$ and $r=1$. (See also in [19, Vol. I, pp. 240-243].)

We recall that the integral modulus of smoothness (in the norm of $L^{p}$ ) of a function $f \in L^{p}(\mathbb{T})$ for some $1 \leq p<\infty$ is defined by $\omega_{2}(f ; \delta)_{p}:=\sup \left\{\left(\frac{1}{2 \pi} \int_{\mathbb{T}}|f(x+h)+f(x-h)-2 f(x)|^{p} d x\right)^{1 / p}: 0<h \leq \delta\right\} ;$

while the ordinary modulus of smoothness of a function $f \in C(\mathbb{T})$ is defined by

$$
\omega_{2}(f ; \delta):=\sup \{|f(x+h)+f(x-h)-2 f(x)|: x \in \mathbb{T}, 0<h \leq \delta\}, \delta>0 .
$$

It is worth observing that Theorem 1.1 and Corollary 1.1 remain valid if the modulus $\omega$ of continuity is replaced by the modulus $\omega_{2}$ of smoothness in them. The proof of this assertion hinges on the fact that the Fourier series of the function $f(x+h)-f(x-h)-2 f(x)$ is of the form

$$
\begin{aligned}
& f(x+h)+f(x-h)-2 f(x) \sim \sum_{m \in \mathbb{Z}} \hat{f}(m) e^{i m x}\left[e^{i m h}+e^{-i m h}-2\right]= \\
& \sum_{m \in \mathbb{Z}} \hat{f}(m) e^{i m x}(2 \cos m h-2)=-4 \sum_{m \in \mathbb{Z}} \hat{f}(m) e^{i m x} \sin ^{2} \frac{m h}{2}, \quad x \in \mathbb{T} .
\end{aligned}
$$

Next, we recall the notion of bounded $s$-variation, where $s \in \mathbb{R}_{+}$. Let

$$
\mathcal{P}_{1}:-\pi=x_{0}<x_{1}<x_{2}<\cdots<x_{m}=\pi
$$

be an arbitrary partition of the closed interval $\overline{\mathbb{T}}:=[-\pi, \pi]$. A periodic function $f$ is said to be of bounded s-variation, in symbols; $f \in B V_{s}(\overline{\mathbb{T}})$, if

$$
V_{s}(f):=\sup \sum_{k=1}^{m}\left|f\left(x_{k}\right)-f\left(x_{k-1}\right)\right|^{s}<\infty
$$


where the supremum is extended for all partitions $\mathcal{P}_{1}$ of $\overline{\mathbb{T}}$. We note that Wiener [16] considered the case $s=2$, and called the functions in $B V_{2}(\overline{\mathbb{T}})$ functions of bounded quadratic variation.

It is not difficult to see that

$$
V_{s_{1}}(\overline{\mathbb{T}}) \subset V_{s_{2}}(\overline{\mathbb{T}}) \text { whenever } 0<s_{1}<s_{2}<\infty
$$

Theorem 1.2. Suppose $f \in C(\mathbb{T}) \cap B V_{s}(\overline{\mathbb{T}})$ for some $s \in(0,2)$. If

$$
\gamma=\left\{\gamma_{m}\right\} \in \mathfrak{A}_{2 /(2-r)} \text { for some } r \in(0,2),
$$

then

$$
\sum(\gamma, f)_{r} \leq \kappa C V_{s}^{r / 2}(f) \sum_{\mu=0}^{\infty} 2^{-\mu r} \Gamma_{\mu-1} \omega^{(2-s) r / 2}\left(f ; \frac{\pi}{2^{\mu}}\right),
$$

where $\kappa$ is from (1.1) corresponding to $\alpha:=2 /(2-r)$ and $\Gamma_{\mu}$ is defined in (1.6).

Corollary 1.2. Under the conditions of Theorem 1.2, we have

$$
\sum(\gamma, f)_{r} \leq \kappa C V_{s}^{r / 2}(f) \sum_{m=1}^{\infty} m^{-r} \gamma_{m} \omega^{(2-s) r / 2}\left(f ; \frac{\pi}{m}\right) .
$$

In the case when $\gamma_{m} \equiv 1$ and $r=1$, it follows from Corollary 1.2 that

$$
\sum(1 ; f)_{1}:=\sum_{|m| \geq 1}|\hat{f}(m)| \leq \kappa C V_{s}^{r / 2}(f) \sum_{m=1}^{\infty} \frac{1}{m} \omega^{(2-s) / 2}\left(f ; \frac{\pi}{m}\right) .
$$

In particular, if $f \in C(\mathbb{T}) \cap B V_{s}(\overline{\mathbb{T}})$ for some $s \in(0,2)$ and

$$
\omega\left(f ; \frac{\pi}{m}\right)=O\left(m^{-\alpha}\right) \quad \text { for some } \quad \alpha>0
$$

or more generally, if

$$
\omega\left(f ; \frac{\pi}{m}\right)=O\left(\left(\log \frac{\pi}{m}\right)^{\beta}\right) \quad \text { for some } \beta>\frac{2}{2-s},
$$


then the Fourier series of $f$ is absolutely convergent.

Let $\gamma_{m} \equiv 1$. Corollary 1.2 was proved by Zygmund [17] (see also Salem [9]) in the case when $f \in \operatorname{Lip}(\alpha) \cap B V_{1}(\overline{\mathbb{T}})$ for some $0<\alpha \leq 1 / 2$ and $r=1$; by Waraszkiewicz [15] and Zygmund [17] in the case when $f \in \operatorname{Lip}(\alpha) \cap B V_{1}(\overline{\mathbb{T}})$ for some $0<\alpha \leq 1$ and $r>2 /(\alpha+2)$. (See also in [19, Vol. I, pp. 241-243].)

Finally, the following theorem is also due to Zygmund [18].

Theorem 1.3. If $f$ is absolutely continuous on $\overline{\mathbb{T}}$ and $f^{\prime} \in L^{p}(\mathbb{T})$ for some $p>1$, then

$$
\sum_{m \in \mathbb{Z}}|\hat{f}(m)| \leq|\hat{f}(0)|+C_{p} V^{1 / 2}(f)\left\|f^{\prime}\right\|_{L^{p}}^{1 / 2}
$$

where the constant $C_{p}$ is given by

$$
C_{p}:=\frac{1}{2} \sum_{\mu=1}^{\infty}\left(\frac{\pi}{2^{\mu}}\right)^{1 /\left(2 p^{\prime}\right)}, \quad \frac{1}{p}+\frac{1}{p^{\prime}}=1 .
$$

\subsection{New results on double Fourier series}

Let $f=f(x, y)$ be a complex-valued function periodic with period $2 \pi$ in each variable. We recall that if $f \in L^{1}\left(\mathbb{T}^{2}\right)$, where $\mathbb{T}^{2}:=\mathbb{T} \times \mathbb{T}$, then the double Fourier series of $f$ is given by

$$
f(x, y) \sim \sum_{m \in \mathbb{Z}} \sum_{n \in \mathbb{Z}} \hat{f}(m, n) e^{i(m x+n y)}, \quad(x, y) \in \mathbb{T}^{2},
$$

where the Fourier coefficients $\hat{f}(m, n)$ are defined by

$$
\hat{f}(m, n):=\frac{1}{4 \pi^{2}} \iint_{\mathbb{T}^{2}} f(x, y) e^{-i(m x+n y)} d x d y, \quad(m, n) \in \mathbb{Z}^{2} .
$$


We will introduce the notion of moduli of continuity for functions of two variables. To this end, we use the notation

$$
\begin{gathered}
\Delta_{1,1}\left(f ; x, y ; h_{1}, h_{2}\right):= \\
f\left(x+h_{1}, y+h_{2}\right)-f\left(x, y+h_{2}\right)-f\left(x+h_{1}, y\right)+f(x, y)
\end{gathered}
$$

where $(x, y) \in \mathbb{T}^{2}$ and $h_{1}, h_{2}>0$. The integral modulus of continuity (in the norm of $\left.L^{p}\right)$ of a function $f \in L^{p}\left(\mathbb{T}^{2}\right)$ for some $1 \leq p<\infty$ is defined by

$$
\begin{gathered}
\omega\left(f ; \delta_{1}, \delta_{2}\right)_{p}:=\sup \left\{\left.\left(\frac{1}{4 \pi^{2}} \iint_{\mathbb{T}^{2}} \mid \Delta_{1,1} f ; x, y ; h_{1}, h_{2}\right)\right|^{p} d x d y\right)^{1 / p}, \\
\left.0<h_{1} \leq \delta_{1} \quad \text { and } 0<h_{2} \leq \delta_{2}\right\}, \delta_{1}, \delta_{2}>0 .
\end{gathered}
$$

It is easy to check that if $f \in L^{p}\left(\mathbb{T}^{2}\right)$ for some $1 \leq p<\infty$, then

$$
\omega\left(f ; \delta_{1}, \delta_{2}\right)_{p} \rightarrow 0 \quad \text { as } \max \left\{\delta_{1}, \delta_{2}\right\} \rightarrow 0
$$

In the case when $f \in C\left(\mathbb{T}^{2}\right)$, the modulus of continuity of $f$ is defined analogously:

$$
\begin{gathered}
\omega\left(f ; \delta_{1}, \delta_{2}\right):=\sup \left\{\left|\Delta_{1,1}\left(f ; x, y ; h_{1}, h_{2}\right)\right|:\right. \\
\left.(x, y) \in \mathbb{T}^{2}, 0<h_{1} \leq \delta_{1} \text { and } 0<h_{2} \leq \delta_{2}\right\}, \delta_{1}, \delta_{2}>0 .
\end{gathered}
$$

Analogously to the definition for a single sequence $\left\{\gamma_{m}\right\}$ to be in $\mathfrak{A}_{\alpha}$ (see (1.1) and (1.2)), we say that a double sequence $\gamma=\left\{\gamma_{m n}:(m, n) \in \mathbb{N}_{+}^{2}\right\}$ of nonnegative numbers belongs to the class $\mathfrak{A}_{\alpha}$ for some $\alpha \geq 1$ if the inequality

$$
\left(\sum_{m \in D_{\mu}} \sum_{n \in D_{\nu}} \gamma_{m n}^{\alpha}\right)^{1 / \alpha} \leq \kappa 2^{(\mu+\nu)(1-\alpha) / \alpha} \sum_{m \in D_{\mu-1}} \sum_{n \in D_{\nu-1}} \gamma_{m n}
$$

is satisfied for all $\mu, \nu \geq 0$, where $D_{\mu}$ is defined in (1.2) for $\mu \geq 0$ and we agree to put

$$
D_{-1}:=D_{0}=\{1\}
$$


For instance, if $\mu \geq 1$ and $\nu=0$, then inequality (1.9) is of the form

$$
\left(\sum_{m \in D_{\mu}} \gamma_{m 1}^{\alpha}\right)^{1 / \alpha} \leq \kappa 2^{\mu(1-\alpha) / \alpha} \sum_{m \in D_{\mu-1}} \gamma_{m 1}
$$

It is easy to check that inclusion (1.3) remains valid; and if a double sequence $\gamma=\left\{\gamma_{m n} \geq 0\right\}$ is such that

$\max \left\{\gamma_{m n}: m \in D_{\mu}, n \in D_{\nu}\right\} \leq \kappa \min \left\{\gamma_{m n}: m \in D_{\mu-1}, n \in D_{\nu-1}\right\},(\mu, \nu) \in \mathbb{N}^{2}$

where $\kappa$ is a constant (cf. (1.4)), then $\gamma \in \mathfrak{A}_{\alpha}$ for every $\alpha \geq 1$. Furthermore, every double sequence $\gamma=\left\{\gamma_{m n}\right\}$ of the form

$$
\gamma_{m n}:=m^{\beta_{1}} n^{\beta_{2}} w_{1}(m) w_{2}(n), \quad(m, n) \in \mathbb{N}_{+}^{2},
$$

belongs to $\mathfrak{A}_{\alpha}$ for every $\alpha \geq 1$, where $\beta_{1}, \beta_{2} \in \mathbb{R}$ and the $w_{j}: \mathbb{R}_{+} \rightarrow \mathbb{R}_{+}$are slowly varying functions (see (1.5)) for $j=1,2$.

For convenience in writing, we agree to put

$$
\gamma_{-m, n}=\gamma_{m,-n}=\gamma_{-m,-n}:=\gamma_{m n}, \quad(m, n) \in \mathbb{N}_{+}^{2} .
$$

After these preliminaries, our first main result reads as follows.

Theorem 1.4. Suppose $f \in L^{p}\left(\mathbb{T}^{2}\right)$ for some $1<p \leq 2$. If

$$
\gamma=\left\{\gamma_{m n}\right\} \in \mathfrak{A}_{p /(p-r p+r)} \text { for some } r \in(0, q),
$$

where $1 / p+1 / q=1$, then

$$
\begin{gathered}
\sum(\gamma ; f)_{r}:=\sum_{|m| \geq 1} \sum_{|n| \geq 1} \gamma_{m n}|\hat{f}(m, n)|^{r} \leq \\
\leq \kappa C \sum_{\mu=0}^{\infty} \sum_{\nu=0}^{\infty} 2^{-(\mu+\nu) r / q} \Gamma_{\mu-1, \nu-1} \omega^{r}\left(f ; \frac{\pi}{2^{\mu}}, \frac{\pi}{2^{\nu}}\right)_{p},
\end{gathered}
$$


where $\kappa$ is from (1.9) corresponding to $\alpha:=p /(p-r p+r)$,

$$
\Gamma_{\mu \nu}:=\sum_{m \in D_{\mu}} \sum_{n \in D_{\nu}} \gamma_{m n} \text { for } \mu, \nu \geq-1
$$

with the agreement (1.10) that

$$
\Gamma_{-1, \nu}:=\Gamma_{0 \nu}, \Gamma_{\mu,-1}:=\Gamma_{\mu 0} \text { for } \mu, \nu \geq 0, \text { and } \Gamma_{-1,-1}:=\Gamma_{00}=\left\{\gamma_{11}\right\}
$$

Proof. Let $h_{1}, h_{2}>0$ be given. Clearly, the Fourier series of

$$
\begin{gathered}
\Delta_{1}\left(f ; x, y ; h_{1}, h_{2}\right):=f\left(x+h_{1}, y+h_{2}\right)-f\left(x-h_{1}, y+h_{2}\right)- \\
f\left(x+h_{1}, y-h_{2}\right)+f\left(x-h_{1}, y-h_{2}\right)
\end{gathered}
$$

is given by

$$
\begin{gathered}
\Delta_{1}\left(f ; x, y ; h_{1}, h_{2}\right) \sim \sum_{m \in \mathbb{Z}} \sum_{n \in \mathbb{Z}} \hat{f}(m, n) e^{i(m x+n y)} \times \\
\times\left[e^{i\left(m h_{1}+n h_{2}\right)}-e^{i\left(-m h_{1}+n h_{2}\right)}-e^{i\left(m h_{1}-n h_{2}\right)}+e^{i\left(-m h_{1}-n h_{2}\right)}\right]= \\
=-4 \sum_{m \in \mathbb{Z}} \sum_{n \in \mathbb{Z}} \hat{f}(m, n) e^{i(m x+n y)} \sin m h_{1} \sin n h_{2}, \quad(x, y) \in \mathbb{T}^{2} .
\end{gathered}
$$

Since $f \in L^{p}\left(\mathbb{T}^{2}\right)$ for some $1<p \leq 2$, we may apply the Hausdorff-Young inequality (see, for example in [19, p. 178, where it is formulated in terms of Fourier transform]), to obtain the inequality

$$
\begin{gathered}
4\left(\sum_{m \in \mathbb{Z}} \sum_{n \in \mathbb{Z}}\left|\hat{f}(m, n) \sin m h_{1} \sin n h_{2}\right|^{q}\right)^{1 / q} \leq \\
\leq\left(\frac{1}{4 \pi^{2}} \iint_{\mathbb{T}^{2}}\left|\Delta_{1}\left(f ; x, y ; h_{1}, h_{2}\right)\right|^{p} d x d y\right)^{1 / p}, \quad \text { where } \frac{1}{p}+\frac{1}{q}=1 .
\end{gathered}
$$

Hence it follows that

$$
\sum_{m \in \mathbb{Z}} \sum_{n \in \mathbb{Z}}\left|\hat{f}(m, n) \sin m h_{1} \sin n h_{2}\right|^{q} \leq 4^{-q} \omega^{q}\left(f ; 2 h_{1}, 2 h_{2}\right)_{p}
$$


Setting

$$
h_{1}:=\frac{\pi}{2^{\mu+1}} \quad \text { and } \quad h_{2}:=\frac{\pi}{2^{\nu+1}}, \quad \mu, \nu \in \mathbb{N},
$$

taking into account that

$$
\frac{\pi}{4}<\frac{|m| \pi}{2^{\mu+1}} \leq \frac{\pi}{2} \quad \text { for } \quad|m| \in D_{\mu}
$$

and an analogous inequality for $|n| \in D_{\nu}$, we obtain

$$
\sum_{|m| \in D_{\mu}} \sum_{|n| \in D_{\nu}}|\hat{f}(m, n)|^{q} \leq 2^{-q} \omega^{q}\left(f ; \frac{\pi}{2^{\mu}}, \frac{\pi}{2^{\nu}}\right)_{p} .
$$

Applying Hölder's inequality with the exponents

$$
\frac{q}{r}=\frac{p}{r(p-1)} \quad \text { and } \quad \frac{q}{q-r}=\frac{p}{p-r p+r},
$$

it follows from (1.11) and (1.17) that

$$
S_{\mu \nu}:=\sum_{|m| \in D_{\mu}} \sum_{|n| \in D_{\nu}} \gamma_{m n}|\hat{f}(m, n)|^{r} \leq
$$

$$
\begin{gathered}
\leq\left(\sum_{|m| \in D_{\mu}} \sum_{|n| \in D_{\nu}}|\hat{f}(m, n)|^{q}\right)^{r / q}\left(\sum_{|m| \in D_{\mu}} \sum_{|n| \in D_{\nu}} \gamma_{m n}^{p /(p-r p+r)}\right)^{(p-r p+r) / p} \leq \\
\leq 2^{-r} \omega^{r}\left(f ; \frac{\pi}{2^{\mu}}, \frac{\pi}{2^{\nu}}\right)_{p}\left(4 \sum_{m \in D_{\mu}} \sum_{n \in D_{\nu}} \gamma_{m n}^{p /(p-r p+r)}\right)^{(p-r p+r) / p} .
\end{gathered}
$$

If $\max \{\mu, \nu\} \geq 1$, then we make use of (1.9) with $\alpha:=p /(p-r p+r)$ and (1.18) to obtain

$$
\begin{aligned}
S_{\mu \nu} & \leq \kappa 2^{-r} \omega^{r}\left(f ; \frac{\pi}{2^{\mu}}, \frac{\pi}{2^{\nu}}\right)_{p} 4^{(p-r p+r) / p} 2^{-(\mu+\nu) r / p} \Gamma_{\mu-1, \nu-1}= \\
& =\kappa 2^{2-3 r+2 r / p} 2^{-(\mu+\nu) r / q} \Gamma_{\mu-1, \nu-1} \omega^{r}\left(f ; \frac{\pi}{2^{\mu}}, \frac{\pi}{2^{\nu}}\right)_{p} .
\end{aligned}
$$

If $\mu=\nu=0$, then from (1.10) and (1.18) it follows immediately that

$$
S_{00}:=\gamma_{11}\left(|\hat{f}(1,1)|^{r}+|\hat{f}(-1,1)|^{r}+|\hat{f}(1,-1)|^{r}+|\hat{f}(-1,-1)|^{r}\right) \leq
$$




$$
\leq 2^{2-3 r+2 r / p} \gamma_{11} \omega^{r}(f ; \pi, \pi)
$$

Taking into account that

$$
\sum_{|m| \geq 1} \sum_{|n| \geq 1} \gamma_{m n}|\hat{f}(m, n)|^{r}=\sum_{\mu=0}^{\infty} \sum_{\nu=0}^{\infty} S_{\mu \nu}
$$

and combining (1.19) and (1.20) yields (1.12) to be proved, with the constant $C:=2^{-r} \cdot 4^{(p-r p+r) / p}$.

Corollary 1.3. Under the conditions of Theorem 1.4, we have

$$
\sum(\gamma ; f)_{r} \leq \kappa C \sum_{m=1}^{\infty} \sum_{n=1}^{\infty}(m n)^{-r / q} \gamma_{m n} \omega^{r}\left(f ; \frac{\pi}{m}, \frac{\pi}{n}\right)_{p} .
$$

Proof. In case $\mu, \nu \geq 1$, from (1.9) and (1.13) it follows that

$$
\begin{gathered}
2^{-(\mu+\nu) r / q} \Gamma_{\mu-1, \nu-1} \omega^{r}\left(f ; \frac{\pi}{2^{\mu}}, \frac{\pi}{2^{\nu}}\right)_{p} \leq \\
\leq \sum_{m \in D_{\mu-1}} \sum_{n \in D_{\nu-1}}(m n)^{-r / q} \gamma_{m n} \omega^{r}\left(f ; \frac{\pi}{m}, \frac{\pi}{n}\right)_{p} .
\end{gathered}
$$

In case $\mu \geq 1$ and $\nu=0$, from (1.10) and (1.13) it follows that

$$
2^{-\mu r / q} \Gamma_{\mu-1, \nu-1} \omega^{r}\left(f ; \frac{\pi}{2^{\mu}}, \pi\right)_{p} \leq \sum_{m \in D_{\mu-1}} m^{-r / q} \gamma_{m 1} \omega^{r}\left(f ; \frac{\pi}{m}, \pi\right)_{p} .
$$

In case $\mu=0$ and $\nu \geq 1$, an analogous inequality holds; while in case $\mu=\nu=0$, we trivially have

$$
\Gamma_{-1,-1} \omega^{r}(f ; \pi, \pi)_{p}=\gamma_{11} \omega^{r}(f ; \pi, \pi)_{p}
$$

Combining these inequalities with (1.12) gives (1.22) to be proved.

We recall that a function $f \in C\left(\mathbb{T}^{2}\right)$ is said to belong to the multiplicative Lipschitz class $\operatorname{Lip}\left(\alpha_{1}, \alpha_{2}\right)$ for some $\alpha_{1}, \alpha_{2}>0$ if

$$
\omega\left(f ; \delta_{1}, \delta_{2}\right)=O\left(\delta_{1}^{\alpha_{1}} \delta_{2}^{\alpha_{2}}\right)
$$


It is worth formulating Theorem 1.4 in the particular case when $f \in$ $\operatorname{Lip}\left(\alpha_{1}, \alpha_{2}\right)$ and $\gamma_{m n} \equiv 1$.

Corollary 1.4. Suppose $f \in \operatorname{Lip}\left(\alpha_{1}, \alpha_{2}\right)$ for some $\alpha_{1}, \alpha_{2}>0$ and $1<p \leq 2$. If

$$
\frac{q}{1+q \min \left\{\alpha_{1}, \alpha_{2}\right\}}<r<q
$$

then

$$
\sum_{|m| \geq 1} \sum_{|n| \geq 1}|\hat{f}(m, n)|^{r}<\infty
$$

Proof. By hypothesis,

$$
\Gamma_{\mu}=2^{\mu} \quad, \Gamma_{\nu}=2^{\nu} \quad \text { and } \omega\left(f ; 2^{-\mu}, 2^{-\nu}\right)_{p} \leq C 2^{-\mu \alpha_{1}-\nu \alpha_{2}}, \quad \mu, \nu \in \mathbb{N} \text {, }
$$

where $C$ is a constant. It follows from (1.12) that

$$
\begin{gathered}
\sum_{|m|>1} \sum_{|n|>1}|\hat{f}(m, n)|^{r} \\
\leq \kappa 4^{-r} C \sum_{\mu=0}^{\infty} \sum_{\nu=0}^{\infty} 2^{-(\mu+\nu) r / q} 2^{\mu+\nu-2} 2^{-\left(\mu \alpha_{1}+\nu \alpha_{2}\right) r} .
\end{gathered}
$$

By (1.24), we have

$$
q<r\left(1+q \alpha_{j}\right) \quad \text { or equivalently } \quad 1<\frac{r}{q}+\alpha_{j} r, \quad j=1,2 .
$$

Therefore, both geometric series on the right-hand side of (1.26) converge. This proves (1.25).

Next, we formulate Theorem 1.4 in the particular case when $\gamma \equiv m^{\beta_{1}} n^{\beta_{2}}$ and $r=1$. 
Corollary 1.5. Suppose $f \in \operatorname{Lip}\left(\alpha_{1}, \alpha_{2}\right)$ for some $\alpha_{1}, \alpha_{2}>0$ and $1<p \leq 2$. If $\beta_{1}, \beta_{2} \in \mathbb{R}$ are such that

$$
\beta_{j}<\alpha_{j}-\frac{1}{p}, \quad j=1,2,
$$

then

$$
\sum_{|m|>1} \sum_{|m|>1} m^{\beta_{1}} n^{\beta_{2}}|\hat{f}(m, n)|<\infty
$$

Proof. Since $\left\{m^{\beta_{1}} n^{\beta_{2}}\right\} \in \mathfrak{A}_{\alpha}$ for all $\beta_{1}, \beta_{2} \in \mathbb{R}$ and $\alpha \geq 1$, by hypothesis, we have

$$
\Gamma_{\mu-1, \nu-1} \leq C 2^{\left(\beta_{1}+1\right) \mu+\left(\beta_{2}+1\right) \nu}
$$

and

$$
\omega\left(f ; 2^{-\mu}, 2^{-\nu}\right) \leq C 2^{-\left(\alpha_{1} \mu+\alpha_{2} \nu\right)}, \quad \mu, \nu \in \mathbb{N},
$$

where $C$ is a constant. It follows again from (1.12) that

$$
\begin{gathered}
\sum_{|m|>1}^{\infty} \sum_{|n|>1}^{\infty} m^{\beta_{1}} n^{\beta_{2}}|\hat{f}(m, n)| \\
\leq \kappa 4^{-r} C^{2} \sum_{\mu=0}^{\infty} \sum_{\nu=0}^{\infty} 2^{-(\mu+\nu) / q} 2^{\left(\beta_{1}+1\right) \mu+\left(\beta_{2}+1\right) \nu} 2^{-\left(\alpha_{1} \mu+\alpha_{2} \nu\right)}, \quad \mu, \nu \in \mathbb{N} .
\end{gathered}
$$

By (1.27), we have

$$
\beta_{j}-\alpha_{j}+1-\frac{1}{q}=\beta_{j}-\alpha_{j}+\frac{1}{p}<0, \quad j=1,2 .
$$

Consequently, both geometric series on the right-hand side of (1.30) converge. This proves (1.28).

Now, Theorem 1.4 and Corollary 1.3 were proved in [7] in the case when $\lambda_{m n} \equiv 1, p=2$ and $r=1$. In particular, in this case the double series on the right-hand side of (1.12) is convergent if

$$
f \in \operatorname{Lip}\left(\alpha_{1}, \alpha_{2}\right) \quad \text { for some } \quad \alpha_{1}, \alpha_{2}>1 / 2 \text {. }
$$


Given a function $f=f(x, y)$ and $h_{1}, h_{2}>0$, we put

$$
\begin{aligned}
& \Delta_{2}\left(f ; x, y ; h_{1}, h_{2}\right):=f\left(x+h_{1}, y+h_{2}\right)+f\left(x-h_{1}, y+h_{2}\right)+ \\
& +f\left(x+h_{1}, y-h_{2}\right)+f\left(x-h_{1}, y-h_{2}\right)-2\left[f\left(x+h_{1}, y\right)+\right. \\
& \left.\quad+f\left(x-h_{1}, y\right)+f\left(x, y+h_{2}\right)+f\left(x, y-h_{2}\right)\right]+4 f(x, y)
\end{aligned}
$$

and define the integral modulus of smoothness (in the norm of $L^{p}$ ) of a function $f \in L^{p}\left(\mathrm{~T}^{2}\right)$ for some $1 \leq p<\infty$ by

$$
\begin{aligned}
\omega_{2}\left(f ; \delta_{1}, \delta_{2}\right)_{p}:=\sup \left\{\left(\frac{1}{4 \pi^{2}} \iint_{\mathbb{T}^{2}}\left|\Delta_{2}\left(f ; x, y ; h_{1}, h_{2}\right)\right|^{p} d x d y\right)^{1 / p}:\right. \\
\left.0<h_{1} \leq \delta_{1}, 0<h_{2} \leq \delta_{2}\right\} ;
\end{aligned}
$$

while we define the modulus of smoothness of a function $f \in C\left(\mathrm{~T}^{2}\right)$ by

$\omega_{2}\left(f ; \delta_{1}, \delta_{2}\right):=\sup \left\{\left|\Delta_{2}\left(f ; x, y ; h_{1}, h_{2}\right)\right|: 0<h_{1} \leq \delta_{1}, 0<h_{2} \leq \delta_{2}\right\}, \delta_{1}, \delta_{2}>0$.

It is worth observing that Theorem 1.4 and Corollary 1.3 remain valid if the modulus of continuity is replaced by the multiplicative modulus of smoothness in them. The proof of this assertion hinges on the fact that the double Fourier series of the function $\Delta_{2}\left(f ; x, y ; h_{1}, h_{2}\right)$ is of the form

$$
\begin{gathered}
\Delta_{2}\left(f ; x, y ; h_{1}, h_{2}\right) \sim \\
\sim \sum_{m \in \mathbb{Z}} \sum_{n \in \mathbb{Z}} \hat{f}(m, n) e^{i(m x+n y)}\left[e^{i m h_{1}}+e^{-i m h_{1}}-2\right]\left[e^{i n h_{2}}+e^{-i n h_{2}}-2\right]= \\
=16 \sum_{m \in \mathbb{Z}} \sum_{n \in \mathbb{Z}} \hat{f}(m, n) e^{i(m x+n y)} \sin ^{2} \frac{m h_{1}}{2} \sin ^{2} \frac{n h_{2}}{2},(x, y) \in \mathbb{T}^{2} .
\end{gathered}
$$

We also note that

$$
\omega_{2}\left(f ; \delta_{1}, \delta_{2}\right) \leq 4 \omega\left(f ; \delta_{1}, \delta_{2}\right)
$$


due to the fact that

$$
\begin{gathered}
\Delta_{2}\left(f ; x, y ; h_{1}, h_{2}\right)= \\
=\left[f\left(x+h_{1}, y+h_{2}\right)-f\left(x, y+h_{2}\right)-f\left(x+h_{1}, y\right)+f(x, y)\right]+ \\
+\left[f\left(x-h_{1}, y+h_{2}\right)-f\left(x, y+h_{2}\right)-f\left(x-h_{1}, y\right)+f(x, y)\right]+ \\
+\left[f\left(x+h_{1}, y-h_{2}\right)-f\left(x, y-h_{2}\right)-f\left(x+h_{1}, y\right)+f(x, y)\right]+ \\
+\left[f\left(x-h_{1}, y-h_{2}\right)-f\left(x, y-h_{2}\right)-f\left(x-h_{1}, y\right)+f(x, y)\right]= \\
=\Delta_{1,1}\left(f ; x, y ; h_{1}, h_{2}\right)+\Delta_{1,1}\left(f ; x-h_{1}, y ; h_{1}, h_{2}\right)+ \\
+\Delta_{1,1}\left(f ; x, y-h_{2} ; h_{1}, h_{2}\right)+\Delta_{1,1}\left(f ; x-h_{1}, y-h_{2} ; h_{1}, h_{2}\right) .
\end{gathered}
$$

A function $f \in C\left(\mathbb{T}^{2}\right)$ is said to belong to the multiplicative Zygmund class $\operatorname{Zyg}\left(\alpha_{1}, \alpha_{2}\right)$ for some $\alpha_{1}, \alpha_{2}>0$ if condition (1.23) is satisfied with $\omega_{2}\left(f ; \delta_{1}, \delta_{2}\right)$ in place of $\omega\left(f ; \delta_{1}, \delta_{2}\right)$. The inclusion

$$
\operatorname{Lip}\left(\alpha_{1}, \alpha_{2}\right) \subset \operatorname{Zyg}\left(\alpha_{1}, \alpha_{2}\right)
$$

follows immediately from inequality (1.31).

Next, we recall the notion of bounded $s$-variation (in the sense of Vitali) for functions of two variables, where $s \in \mathbb{R}_{+}$. We consider an arbitrary partition $\mathcal{P}=\mathcal{P}_{1} \times \mathcal{P}_{2}$ of the closed square $\overline{\mathbb{T}}^{2}$, where $\mathcal{P}_{1}$ is given in (1.7) and $\mathcal{P}_{2}$ is given by

$$
\mathcal{P}_{2}:-\pi=y_{0}<y_{1}<y_{2}<\cdots<y_{n}=\pi
$$

A function $f=f(x, y)$ periodic in each variable with period $2 \pi$ is said to be of bounded $s$-variation, in symbols: $f \in B V_{s}\left(\overline{\mathbb{T}}^{2}\right)$, if

$$
\begin{gathered}
V_{s}(f):=\sup \sum_{k=1}^{m} \sum_{\ell=1}^{n} \mid f\left(x_{k}, y_{\ell}\right)-f\left(x_{k-1}, y_{\ell}\right)- \\
-f\left(x_{k}, y_{\ell-1}\right)+\left.f\left(x_{k-1}, y_{\ell-1}\right)\right|^{s}<\infty
\end{gathered}
$$


where the supremum is extended for all partitions $\mathcal{P}$ of $\overline{\mathbb{T}}^{2}$. In the case when $s=1$, see this definition for example in [3].

Now, our second main result reads as follows.

Theorem 1.5. Suppose $f \in C\left(\mathbb{T}^{2}\right) \cap B V_{s}\left(\overline{\mathbb{T}}^{2}\right)$ for some $s \in(0,2)$. If

$$
\gamma=\left\{\gamma_{m n}\right\} \in \mathfrak{A}_{2 /(2-r)} \text { for some } r \in(0,2)
$$

then

$$
\begin{gathered}
\sum(\gamma ; f)_{r}:=\sum_{|m| \geq 1} \sum_{|n| \geq 1} \gamma_{m n}|\hat{f}(m, n)|^{r} \leq \\
\leq \kappa C V_{s}^{r / 2}(f) \sum_{\mu=0}^{\infty} \sum_{\nu=0}^{\infty} 2^{-(\mu+\nu) r} \Gamma_{\mu-1, \nu-1} \omega^{(2-s) r / 2}\left(f ; \frac{\pi}{2^{\mu}}, \frac{\pi}{2^{\nu}}\right),
\end{gathered}
$$

where $\kappa$ is from (1.9) corresponding to $\alpha:=2 /(2-r), V_{s}(f)$ is defined in (1.32), and $\Gamma_{\mu \nu}$ is defined in (1.13) and (1.14).

Proof. By the assumption of continuity and bounded $s$-variation, we have

$$
\begin{gathered}
\sum_{k=1}^{2 M} \sum_{\ell=1}^{2 N} \mid f\left(x+\frac{k \pi}{M}, y+\frac{\ell \pi}{N}\right)-f\left(x+\frac{(k-1) \pi}{M}, y+\frac{\ell \pi}{N}\right)- \\
-f\left(x+\frac{k \pi}{M}, y+\frac{(\ell-1) \pi}{N}\right)+\left.f\left(x+\frac{(k-1) \pi}{M}, y+\frac{(\ell-1) \pi}{N}\right)\right|^{2} \leq \\
\leq \omega^{2-s}\left(f ; \frac{\pi}{M}, \frac{\pi}{N}\right) V_{s}(f) \text { for all integers } M, N \geq 1 .
\end{gathered}
$$

We integrate both sides of this inequality over $\mathbb{T}^{2}$ with respect to $x$ and $y$, and observe that each of the integrals on the left-hand side in (1.34) is equal to the following one:

$$
I_{M N}:=\iint_{\mathbb{T}^{2}} \mid f\left(x+\frac{\pi}{2 M}, y+\frac{\pi}{2 N}\right)-f\left(x-\frac{\pi}{2 M}, y+\frac{\pi}{2 N}\right)-
$$




$$
-f\left(x+\frac{\pi}{2 M}, y-\frac{\pi}{2 N}\right)+\left.f\left(x-\frac{\pi}{2 M}, y-\frac{\pi}{2 N}\right)\right|^{2} d x d y .
$$

As a result, we obtain the inequality

$$
I_{M N} \leq \frac{\pi^{2}}{M N} \omega^{2-s}\left(f ; \frac{\pi}{M}, \frac{\pi}{N}\right) V_{s}(f) .
$$

Applying the Parseval inequality (applied to the double Fourier series in (1.15) with $h_{1}:=\pi / 2 M$ and $\left.h_{2}:=\pi / 2 N\right)$ gives

$$
\frac{1}{4 \pi^{2}} I_{M N}=16 \sum_{m \in \mathbb{Z}} \sum_{n \in \mathbb{Z}}\left|\hat{f}(m, n) \sin \frac{m \pi}{2 M} \sin \frac{n \pi}{2 N}\right|^{2} .
$$

Combining (1.35) and (1.36) yields

$$
\begin{gathered}
\sum_{m \in \mathbb{Z}} \sum_{n \in \mathbb{Z}}\left|\hat{f}(m, n) \sin \frac{m \pi}{2 M} \sin \frac{n \pi}{2 N}\right|^{2}=\frac{1}{64 \pi^{2}} I_{m n} \leq \\
\leq \frac{1}{64 M N} \omega^{2-s}\left(f ; \frac{\pi}{M}, \frac{\pi}{N}\right) V_{s}(f) .
\end{gathered}
$$

We put

$$
M:=2^{\mu} \quad \text { and } \quad N:=2^{\nu}, \quad \mu, \nu \in \mathbb{N}
$$

in this inequality and take into account (1.16) to obtain

$$
\sum_{|m| \in D_{\mu}} \sum_{|n| \in D_{\nu}}|\hat{f}(m, n)|^{2} \leq 2^{-4} 2^{-\mu} 2^{-\nu} \omega^{2-s}\left(f ; \frac{\pi}{2^{\mu}}, \frac{\pi}{2^{\nu}}\right) V_{s}(f) .
$$

Applying Hölder's inequality with the exponents $2 / r$ and $2 /(2-r)$, hence it follows that

$$
S_{\mu \nu}:=\sum_{|m| \in D_{\mu}} \sum_{|n| \in D_{\nu}} \gamma_{m n}|\hat{f}(m, n)|^{r} \leq
$$

$$
\leq\left(\sum_{|m| \in D_{\mu}} \sum_{|n| \in D_{\nu}}|\hat{f}(m, n)|^{2}\right)^{r / 2}\left(\sum_{|m| \in D_{\mu}} \sum_{|n| \in D_{\nu}} \gamma_{m n}^{2 /(2-r)}\right)^{(2-r) / 2} \leq
$$

$\leq 2^{-2 r} 2^{-(\mu+\nu) r / 2} \omega^{(2-s) r / 2}\left(f ; \frac{\pi}{2^{\mu}}, \frac{\pi}{2^{\nu}}\right) V_{s}^{r / 2}(f)\left(4 \sum_{m \in D_{\mu}} \sum_{n \in D_{\nu}} \gamma_{m n}^{2 /(2-r)}\right)^{(2-r) / 2}$. 
In case $\max \{\mu, \nu\} \geq 1$, we make use of (1.9) with $\alpha:=2 /(2-r)$ and (1.37) to find that

$$
\begin{gathered}
S_{\mu \nu} \leq 2^{-2 r} 2^{-(\mu+\nu) r / 2} V_{s}^{r / 2}(f) \times \\
\times \omega^{(2-s) r / 2}\left(f ; \frac{\pi}{2^{\mu}}, \frac{\pi}{2^{\nu}}\right) 4^{(2-r) / 2} \kappa 2^{-(\mu+\nu) r / 2} \Gamma_{\mu-1, \nu-1}= \\
=\kappa 2^{2-3 r} V_{s}^{r / 2}(f) 2^{-(\mu+\nu) r} \Gamma_{\mu-1, \nu-1} \omega^{(2-s) r / 2}\left(f ; \frac{\pi}{2^{\mu}}, \frac{\pi}{2^{\nu}}\right) .
\end{gathered}
$$

In case $\mu=\nu=0$, from (1.37) it follows immediately that (cf. (1.20))

$$
S_{00} \leq 2^{2-3 r} V_{s}^{r / 2}(f) \gamma_{11} \omega^{(2-s) r / 2}(f ; \pi, \pi)
$$

Taking into account (1.21), and putting together (1.38) and (1.39) give (1.33) to be proved, with the constant $C:=2^{2-3 r}$.

Corollary 1.6. Under the conditions of Theorem 1.4, we have

$$
\sum(\gamma ; f)_{r} \leq \kappa C V_{s}^{r / 2}(f) \sum_{m=1}^{\infty} \sum_{n=1}^{\infty}(m n)^{-r} \gamma_{m n} \omega^{(2-s) r / 2}\left(f ; \frac{\pi}{m}, \frac{\pi}{n}\right) .
$$

Proof. It is analogous to the proof of Corollary 3.

We formulate Theorem 1.5 in the particular case when the function $f \in$ $B V_{s}\left(\overline{\mathbb{T}}^{2}\right) \cap \operatorname{Lip}\left(\alpha_{1}, \alpha_{2}\right)$ and $\gamma \equiv 1$.

Corollary 1.7. Suppose $f \in \operatorname{Lip}\left(\alpha_{1}, \alpha_{2}\right) \cap B V_{s}\left(\overline{\mathbb{T}}^{2}\right)$ for some $\alpha_{1}, \alpha_{2}>0$ and $0<s<2$. If

$$
r>\frac{1}{1+(1-s / 2) \min \left\{\alpha_{1}, \alpha_{2}\right\}},
$$

then (1.25) is satisfied.

Proof. Let $\mu, \nu \in \mathbb{N}$. By hypothesis, we have

$$
\Gamma_{\mu-1, \nu-1}=2^{\mu+\nu-2} \text { and } \omega\left(f ; 2^{-\mu}, 2^{-\nu}\right) \leq C 2^{-\mu \alpha_{1}-\nu \alpha_{2}},
$$


where $C$ is a constant. It follows from (1.33) that

$$
\begin{aligned}
& \sum_{|m|>1}^{\infty} \sum_{|n|>1}^{\infty}|\hat{f}(m, n)|^{r} \leq \kappa 4^{-r} C\left|V_{s}(f)\right|^{r s / 2} \times \\
& \times \sum_{\mu=0}^{\infty} \sum_{\nu=0}^{\infty} 2^{-(\mu+\nu) r} 2^{\mu+\nu-2} 2^{-\left(\mu \alpha_{1}+\nu \alpha_{2}\right)(2-s) r / 2} .
\end{aligned}
$$

By (1.41), we have

$$
-r+1-\alpha_{j}(2-s) r / 2<0, \quad j=1,2 .
$$

Therefore, both geometric series on the right-hand side of (1.42) converge. This proves (1.25).

Finally, we formulate Theorem 1.5 in the special case when $r=1$ and $\gamma_{m n}=m^{\beta_{1}} n^{\beta_{2}}$, where $\beta_{1}, \beta_{2} \in \mathbb{R}$.

Corollary 1.8. Suppose $f \in \operatorname{Lip}\left(\alpha_{1}, \alpha_{2}\right) \cap B V_{s}\left(\overline{\mathbb{T}}^{2}\right)$ for some $\alpha_{1}, \alpha_{2}>0$ and $0<s<2$. If

$$
\beta_{j}<(1-s / 2) \alpha_{j}, \quad j=1,2,
$$

then (1.28) is satisfied.

Proof. Let $\mu, \nu \in \mathbb{N}$. By hypothesis, we have

$$
\Gamma_{\mu-1, \nu-1} \leq C 2^{\left(\beta_{1}+1\right) \mu+\left(\beta_{2}+1\right) \nu} \quad \text { and } \omega\left(f ; 2^{-\mu}, 2^{-\nu}\right)_{p} \leq C 2^{-\left(\alpha_{1} \mu+\alpha_{2} \nu\right)},
$$

where $C$ is a constant. It follows from (1.33) that

$$
\begin{aligned}
& \sum_{|m| \geq 1} \sum_{|n| \geq 1} m^{\beta_{1}} n^{\beta_{2}}|\hat{f}(m, n)| \leq \kappa 4^{-1}\left|V_{s}(f)\right|^{s / 2} C^{2} \times \\
& \times \sum_{\mu=0}^{\infty} \sum_{\nu=0}^{\infty} 2^{-\mu-\nu} 2^{\left(\beta_{1}+1\right) \mu+\left(\beta_{2}+1\right) \nu} 2^{-\left(\alpha_{1} \mu+\alpha_{2} \nu\right)(2-s) / 2} .
\end{aligned}
$$


Due to (1.43), both geometric series on the right-hand side of (1.44) converge. This proves (1.28).

Theorem 1.5 and Corollary 1.4 were proved in [7] in the case when $\gamma_{m n} \equiv$ $1, s=1$ and $r=1$. More generally, in this particular case the double series on the right-hand side of (1.40) is convergent if

$$
\omega\left(f ; \frac{\pi}{m}, \frac{\pi}{n}\right)=O\left(\left(\log \frac{\pi}{m}\right)^{\beta_{1}}\left(\log \frac{\pi}{n}\right)^{\beta_{2}}\right) \quad \text { for some } \quad \beta_{1}, \beta_{2}>\frac{2}{2-s} .
$$

We recall (see, for example, [1]) that a function $f$ defined on a rectangle $R:=[a, b] \times[c, d]$ is said to be absolutely continuous, in symbols: $f \in A C(R)$, if the following two conditions are satisfied:

(i) Given $\varepsilon>0$, there exists $\delta=\delta(\varepsilon)>0$ such that

$$
\sum_{R_{k} \in \mathcal{R}}\left|f\left(b_{k}, d_{k}\right)-f\left(a_{k}, d_{k}\right)-f\left(b_{k}, c_{k}\right)+f\left(a_{k}, c_{k}\right)\right|<\varepsilon
$$

whenever $\mathcal{R}=\left\{R_{k}:=\left[a_{k}, b_{k}\right] \times\left[c_{k}, d_{k}\right]\right\}$ is a finite collection of pairwise nonoverlapping subrectangles of $R$ with

$$
\sum_{R_{k} \in \mathcal{R}}\left(b_{k}-a_{k}\right)\left(d_{k}-c_{k}\right)<\delta .
$$

(ii) The marginal functions $f(\cdot, c)$ and $f(a, \cdot)$ are absolutely continuous as functions of a single variable on the intervals $[a, b]$ and $[c, d]$, respectively.

It is straightforward to check that (i) and (ii) together imply that $f$ is continuous and of bounded variation on the rectangle $R$ in the sense of Hardy and Krause, and each of the marginal functions $f\left(\cdot, y_{0}\right)$ and $f\left(x_{0}, \cdot\right)$ is absolutely continuous in the single variable sense on the intervals $[a, b]$ and $[c, d]$, respectively, where $y_{0} \in[c, d]$ and $x_{0} \in[a, b]$ are fixed. 
The following characterization is proved in [1]: a function $f$ is absolutely continuous on $R:=[a, b] \times[c, d]$ if and only if there exist functions $g \in$ $A C([a, b]), h \in A C([c, d])$ and $\phi \in L^{1}(R)$ such that

$$
f(x, y)=g(x)+h(y)+\int_{a}^{x} \int_{c}^{y} \phi(u, v) d u d v, \quad x \in[a, b], y \in[c, d] .
$$

Clearly, in this case the mixed partial derivative of $f$ exists and

$$
f_{x, y}:=\frac{\partial^{2}}{\partial x \partial y} f(x, y)=\phi(x, y) \quad \text { a.e. }
$$

Now, in the special case when $\gamma_{m n} \equiv 1$ and $r=1$ Theorem 1.5 yields the folloving

Theorem 1.6. If $f \in A C\left(\overline{\mathbb{T}}^{2}\right)$ and $f_{x, y} \in L^{p}\left(\mathbb{T}^{2}\right)$ for some $p>1$, then

$$
\sum_{|m| \geq 1} \sum_{|n| \geq 1}|\hat{f}(m, n)| \leq C_{p} V_{2}^{1 / 2}(f)\left\|f_{x, y}\right\|_{L^{p}}^{1 / 2}
$$

where the constant $C_{p}$ is given by

$$
C_{p}:=\frac{1}{4}\left(\sum_{\mu=1}^{\infty} \frac{\pi}{2^{\mu}}\right)^{1 / p^{\prime}}, \quad \frac{1}{p}+\frac{1}{p^{\prime}}=1 .
$$

Proof. From the assumption $f \in A C\left(\overline{\mathbb{T}}^{2}\right)$ it follows that $f \in B V_{H}\left(\overline{\mathbb{T}}^{2}\right)$. Furthermore, by (1.45), (1.46) and Hölder's inequality, we have

$$
\begin{gathered}
\left|f\left(x+h_{1}, y+h_{2}\right)-f\left(x, y+h_{2}\right)-f\left(x+h_{1}, y\right)+f(x, y)\right| \\
=\left|\int_{x}^{x+h_{1}} \int_{y}^{y+h_{2}} f_{x, y}(u, v) d u d v\right| \\
\leq\left\{\int_{x}^{x+h_{1}} \int_{y}^{y+h_{2}}\left|f_{x, y}(u, v)\right|^{p} d u d v\right\}^{1 / p}\left(h_{1} h_{2}\right)^{1 / p^{\prime}} \\
\leq\left\|f_{x, y}\right\|_{L^{p}}\left(h_{1} h_{2}\right)^{1 / p^{\prime}}, \quad \frac{1}{p}+\frac{1}{p^{\prime}}=1 .
\end{gathered}
$$

Applying Theorem 4 yields (2.7) to be proved. 
Combining Theorems 1.1 and 1.4, Theorems 1.2 and 1.5 or Theorems 1.3 and 1.6, we can easily find sufficient conditions imposed on $f, f_{1}$ and $f_{2}$ for the convergence of the double series

$$
\sum_{m \in \mathbb{Z}} \sum_{n \in \mathbb{Z}} \gamma_{m n}|\hat{f}(m, n)|^{r}
$$

As an illustration, it is worth to present some simple corollaries in the particular case when $\gamma_{m n} \equiv 1$ and $r=1$, these conditions ensure the absolute convergence of the double Fourier series of $f$.

\subsection{Absolute convergence of double Fourier series}

Denote by $\mathcal{A}\left(\mathbb{T}^{2}\right)$ the collection of functions $f \in L^{1}\left(\mathbb{T}^{2}\right)$ whose double Fourier series converge absolutely. It is clear that $f \in \mathcal{A}\left(\mathbb{T}^{2}\right)$ if and only if

$$
\|f\|_{\mathcal{A}\left(\mathbb{T}^{2}\right)}:=\sum_{m=0}^{\infty} \sum_{n=0}^{\infty}|\hat{f}(m, n)|<\infty .
$$

Morever, each $f \in \mathcal{A}\left(\mathbb{T}^{2}\right)$ is a continuous function, since it has a uniformly convergent Fourier series.

We note that $\|\cdot\|_{\mathcal{A}\left(\mathbb{T}^{2}\right)}$ is a norm. Since the mapping $f \rightarrow\{\hat{f}(m, n)$ : $m, n \in \mathbb{N}\}$ is a one-to-one linear isometry from $\mathcal{A}\left(\mathbb{T}^{2}\right)$ to $l^{1}$, it is evident that $\mathcal{A}\left(\mathbb{T}^{2}\right)$ is a Banach space. Similarly to the case of single Fourier series, $\mathcal{A}\left(\mathbb{T}^{2}\right)$ is even a Banach algebra with respect to pointwise multiplication.

If a function $f \in L^{1}\left(\mathbb{T}^{2}\right)$ is such that

$$
\hat{f}(m, n)=0 \quad \text { whenever } \min \{m, n\}=0,
$$

then each of the conditions (1.12) and (1.33) in case $\gamma_{m n} \equiv 1$ and $r=1$, condition (1.25) in case $r=1$, and condition (1.28) in case $\beta_{1}=\beta_{2}=0$ is sufficient to conclude (1.48). 
If condition (1.49) is not satisfied, we may proceed as follows. Observe that

$$
\hat{f}(m, 0)=\hat{f}_{1}(m), \quad \text { where } \quad f_{1}(x):=\frac{1}{2 \pi} \int_{\mathbb{T}} f(x, y) d y, x \in \mathbb{T}
$$

and

$$
\hat{f}(0, n)=\hat{f}_{2}(n), \quad \text { where } \quad f_{2}(x):=\frac{1}{2 \pi} \int_{\mathbb{T}} f(x, y) d x, y \in \mathbb{T} .
$$

By Hölder's inequality, we have $f_{1}, f_{2} \in L^{p}(\mathbb{T})$ whenever $f \in L^{p}\left(\mathbb{T}^{2}\right)$ for some $1 \leq p<\infty$. Analogously to (1.48), we may write that (see [19])

$$
\left\|f_{1}\right\|_{\mathcal{A}(\mathbb{T})}:=\sum_{m=0}^{\infty}\left|\hat{f}_{1}(m)\right|=\sum_{m \in \mathbb{Z}}|\hat{f}(m, 0)|
$$

and

$$
\left\|f_{2}\right\|_{\mathcal{A}(\mathbb{T})}:=\sum_{n=0}^{\infty}\left|\hat{f}_{2}(n)\right|=\sum_{n \in \mathbb{Z}}|\hat{f}(0, n)| .
$$

Combining these with (1.48) gives

$$
\|f\|_{\mathcal{A}\left(\mathbb{T}^{2}\right)}=\sum_{m=1}^{\infty} \sum_{n=1}^{\infty}|\hat{f}(m, n)|+\left\|f_{1}\right\|_{\mathcal{A}(\mathbb{T})}+\left\|f_{2}\right\|_{\mathcal{A}(\mathbb{T})}-|\hat{f}(0,0)| .
$$

Assume $\left\|f_{1}\right\|_{\mathcal{A}(\mathbb{T})}<\infty$ and $\left\|f_{2}\right\|_{\mathcal{A}(\mathbb{T})}<\infty$, then the double Fourier series in (2.6) is absolutely convergent if and only if

$$
\sum_{m=1}^{\infty} \sum_{n=1}^{\infty}|\hat{f}(m, n)|<\infty
$$

Combining Bernstein theorem for single Fourier series with our Corollary 1.3 for $\gamma \equiv 1$ and $r=1$ yields the following

Corollary 1.9. If a function $f: \mathbb{T}^{2} \rightarrow \mathbb{R}$ is such that $f \in \operatorname{Lip}\left(\alpha_{1}, \alpha_{2}\right)$, $f_{1} \in \operatorname{Lip}\left(\alpha_{3}\right)$ and $f_{2} \in \operatorname{Lip}\left(\alpha_{4}\right)$ for some $\alpha_{j}>1 / 2, j=1,2,3,4$; where $f_{1}$ and $f_{2}$ are defined in (1.50) and (1.51), then $f \in \mathcal{A}\left(\mathbb{T}^{2}\right)$. 
Proof. Since $f \in L^{2}\left(\mathbb{T}^{2}\right)$, it is easy to check that $f_{1}, f_{2} \in L^{2}(\mathbb{T})$.

Combining Zygmund theorem for single Fourier series with our Corollary 1.4 for $\gamma \equiv 1, r=1$ and $s=1$ yields the following

Corollary 1.10. If a funtion $f: \mathbb{T}^{2} \rightarrow \mathbb{R}$ is such that $f \in \operatorname{Lip}\left(\alpha_{1}, \alpha_{2}\right) \cap$ $B V_{H}\left(\overline{\mathbb{T}}^{2}\right), f_{1} \in \operatorname{Lip}\left(\alpha_{3}\right) \cap B V(\overline{\mathbb{T}})$ and $f_{2} \in \operatorname{Lip}\left(\alpha_{4}\right) \cap B V(\overline{\mathbb{T}})$ for some $\alpha_{j}>0$, $j=1,2,3,4$ and $0<s, s_{1}, s_{2}<2$; where $f_{1}$ and $f_{2}$ are defined in (1.50) and (1.51), then $f \in \mathcal{A}\left(\mathbb{T}^{2}\right)$.

Proof. Since $f \in B V_{H}\left(\overline{\mathbb{T}}^{2}\right)$, it is easy to check that $f_{1}, f_{2} \in B V(\overline{\mathbb{T}})$.

Combining Theorem 3 and Theorem 6 yields the following

Corollary 1.11. If $f \in A C\left(\overline{\mathbb{T}}^{2}\right), f_{x, y} \in L^{p}\left(\mathbb{T}^{2}\right), g^{\prime} \in L^{p_{1}}(\mathbb{T})$ and $h^{\prime} \in L^{p_{2}}(\mathbb{T})$ for some $p, p_{1}, p_{2}>1$, where the functions $g$ and $h$ occur in (1.45), then the double Fourier series of $f$ converges absolutely.

Proof. Due to Theorem 1.6, it remains to prove that

$$
\sum_{|m| \geq 1}|\hat{f}(m, 0)|+\sum_{|n| \geq 1}|\hat{f}(0, n)|<\infty .
$$

To this effect, we consider the functions $f_{1}$ and $f_{2}$ defined in (1.50) and (1.51), respectively.

Since $f \in B V_{H}\left(\overline{\mathbb{T}}^{2}\right)$, it is easy to check that $f_{1} \in B V(\overline{\mathbb{T}})$. By (1.45) (with $a=c=-\pi$ ), (1.46) and Hölder's inequality, we may estimate as follows:

$$
\begin{aligned}
& |f(x+h)-f(x)| \leq|g(x+h)-g(x)|+\left|\int_{x}^{x+h} \int_{-\pi}^{y} f_{x, y}(u, v) d u d v\right| \\
& \leq\left\|g^{\prime}\right\|_{L^{p_{1}}}|h|^{1 / p_{1}^{\prime}}+\left\|f_{x, y}\right\|_{L^{p}}(2 \pi h)^{1 / p^{\prime}} \leq C|h|^{1 / p_{0}}, \quad p_{0}:=\min \left\{p^{\prime}, p_{1}^{\prime}\right\},
\end{aligned}
$$

where the constant $C$ does not depend on $x, y$ and $h$. Hence it follows immediately that $f_{1} \in \operatorname{Lip}\left(1 / p_{0}\right)$. In an analogous way, we prove that $f_{2} \in$ $B V(\overline{\mathbb{T}}) \cap \operatorname{Lip}\left(1 / p_{0}\right)$. Applying Theorem 1.3 yields (1.52). 


\subsection{Extension to multiple Fourier series}

Let $f=f\left(x_{1}, \ldots, x_{N}\right)$ be a complex-valued function periodic in each variable with period $2 \pi$. We recall (see, e.g. [19, Vol. II. Ch. 17]) that the multiple Fourier series of $f \in L^{1}\left(\mathbb{T}^{N}\right)$ is given by

$$
\begin{gathered}
f\left(x_{1}, \ldots, x_{N}\right) \sim \\
\sum_{m_{1} \in \mathbb{Z}} \ldots \sum_{m_{N} \in \mathbb{Z}} \hat{f}\left(m_{1}, \ldots, m_{N}\right) e^{i\left(m_{1} x_{1}+\cdots+m_{N} x_{N}\right)}, \quad\left(x_{1}, \ldots, x_{N}\right) \in \mathbb{T}^{N},
\end{gathered}
$$

where the Fourier coefficients $\hat{f}\left(m_{1}, \ldots, m_{N}\right)$ are defined by

$$
\begin{gathered}
\hat{f}\left(m_{1}, \ldots, m_{N}\right):=\frac{1}{(2 \pi)^{N}} \int \cdots \int_{\mathbb{T}^{N}} f\left(x_{1}, \ldots, x_{N}\right) \times \\
\times e^{-i\left(m_{1} x_{1}+\cdots+m_{N} x_{N}\right)} d x_{1} \ldots d x_{N}, \quad\left(m_{1}, \ldots, m_{N}\right) \in \mathbb{Z}^{N} .
\end{gathered}
$$

We will introduce the notion of moduli of continuity for functions of several variables. To this effect, we use the notation

$$
\begin{gathered}
\Delta_{1, \ldots, 1}\left(f ; x_{1}, \ldots, x_{N} ; h_{1}, \ldots, h_{N}\right):= \\
=\sum_{\eta_{1}=0}^{1} \ldots \sum_{\eta_{N}=0}^{1}(-1)^{\eta_{1}+\cdots+\eta_{N}} f\left(x_{1}+\eta_{1} h_{1}, \ldots, x_{N}+\eta_{N} h_{N}\right),
\end{gathered}
$$

where $\left(x_{1}, \ldots, x_{N}\right) \in \mathbb{T}^{N}$ and $h_{1}, \ldots, h_{N}>0$. The multiplicative integral modulus of continuity (in the norm of $L^{p}$ ) of a function $f \in L^{p}\left(\mathbb{T}^{N}\right)$ for some $1 \leq p<\infty$ is defined by

$$
\begin{gathered}
\omega\left(f ; \delta_{1}, \ldots, \delta_{N}\right)_{p}:= \\
:=\sup \left\{\left(\frac{1}{(2 \pi)^{N}} \int \ldots \int_{\mathbb{T}^{N}}\left|\Delta_{1, \ldots, 1}\left(f ; x_{1}, \ldots, x_{N} ; h_{1}, \ldots, h_{N}\right)\right|^{p} d x_{1} \ldots d x_{N}\right)^{1 / p}:\right. \\
\left.0<h_{j} \leq \delta_{j}, \quad j=1, \ldots, N\right\}, \quad \delta_{1}, \ldots, \delta_{N}>0 .
\end{gathered}
$$


It is easy to check that for every $f \in L^{p}\left(\mathbb{T}^{N}\right)$,

$$
\omega\left(f ; \delta_{1}, \ldots, \delta_{N}\right)_{p} \rightarrow 0 \quad \text { as } \max \left\{\delta_{1}, \ldots, \delta_{N}\right\} \rightarrow 0
$$

In the case when $f \in C\left(\mathbb{T}^{N}\right)$, the multiplicative modulus of continuity of $f$ is defined by

$$
\begin{gathered}
\omega\left(f ; \delta_{1}, \ldots, \delta_{N}\right):=\sup \left\{\left|\Delta_{1, \ldots, 1}\left(f ; x_{1}, \ldots, x_{N} ; h_{1}, \ldots, h_{N}\right)\right|:\right. \\
\left.\left(x_{1}, \ldots, x_{N}\right) \in \mathbb{T}^{N}, \quad 0<h_{j} \leq \delta_{j}, \quad j=1,2, \ldots, N\right\}, \quad \delta_{1}, \ldots, \delta_{N}>0 .
\end{gathered}
$$

Analogously to (1.1) and (1.9), an $N$-multiple sequence $\gamma=\left\{\gamma_{m_{1}}, \ldots, \gamma_{m_{N}}\right.$ : $\left.\left(m_{1}, \ldots, m_{N}\right) \in \mathbb{N}_{+}^{N}\right\}$ of nonnegative numbers is said to belong to the class $\mathcal{A}_{\alpha}$ for some $\alpha \geq 1$ if there exists a constant $\kappa \geq 1$ such that the inequality

$$
\begin{gathered}
\left(\sum_{m_{1} \in D_{\mu_{1}}} \ldots \sum_{m_{n} \in D_{\mu_{N}}} \gamma_{m_{1}, \ldots, m_{N}}^{\alpha}\right)^{1 / \alpha} \leq \\
\leq \kappa 2^{\left(\mu_{1}+\cdots+\mu_{N}\right)(1-\alpha) / \alpha} \sum_{m_{1} \in D_{\mu_{1}-1}} \ldots \sum_{m_{N} \in D_{\mu_{N}-1}} \gamma_{m_{1}, \ldots, m_{N}}
\end{gathered}
$$

is satisfied for all $\mu_{1}, \ldots, \mu_{N} \in \mathbb{N}$, where $D_{\mu}$ is defined in (1.2) for $\mu \geq 0$ and $D_{-1}:=D_{0}=\{1\}$.

Now, the extensions of Theorem 1.4 and Corollary 1.4 read as follow.

Theorem 1.7. Suppose $f \in L^{p}\left(\mathbb{T}^{N}\right)$ for some $1<p \leq 2$. If

$$
\gamma=\left\{\gamma_{m_{1}, \ldots, m_{N}}\right\} \in \mathfrak{A}_{p /(p-r p+r)} \quad \text { for some } r \in(0, q),
$$

where $1 / p+1 / q=1$, then

$$
\begin{gathered}
\sum(\gamma ; f)_{r}:=\sum_{\left|m_{1}\right| \geq 1} \ldots \sum_{\left|m_{N}\right| \geq 1} \gamma_{m_{1}, \ldots, m_{N}}\left|\hat{f}\left(m_{1}, \ldots, m_{N}\right)\right|^{r} \leq \\
\leq \kappa C \sum_{\mu_{1}=0}^{\infty} \ldots \sum_{\mu_{N}=0}^{\infty} 2^{-\left(\mu_{1}+\cdots+\mu_{N}\right) r / q} \Gamma_{\mu_{1}-1, \ldots, \mu_{N}-1} \omega^{r}\left(f ; \frac{\pi}{2^{\mu_{1}}}, \ldots, \frac{\pi}{2^{\mu_{N}}}\right)_{p}
\end{gathered}
$$


where $\kappa$ is from (1.54) corresponding to $\alpha:=p /(p-r p+r)$ and

$$
\Gamma_{\mu_{1}, \ldots, \mu_{N}}:=\sum_{m_{1} \in D_{\mu_{1}}} \cdots \sum_{m_{N} \in D_{\mu_{N}}} \gamma_{m_{1}, \ldots, m_{N}}
$$

for $\mu_{1}, \ldots, \mu_{N} \geq-1$ (cf. (1.9), (1.10), (1.13) and (1.14)).

Corollary 1.12. Under the conditions of Theorem 1.7, we have

$\sum(\gamma ; f)_{r} \leq \kappa C \sum_{m_{1}=1}^{\infty} \ldots \sum_{m_{N}=1}^{\infty}\left(m_{1} \ldots m_{N}\right)^{-r / q} \gamma_{m_{1}, \ldots, m_{N}} \omega^{r}\left(f ; \frac{\pi}{m_{1}}, \ldots, \frac{\pi}{m_{N}}\right)_{p}$.

A function $f \in C\left(\mathbb{T}^{N}\right)$ is said to belong to the Lipschitz class $\operatorname{Lip}\left(\alpha_{1}, \ldots, \alpha_{N}\right)$ for some $\alpha_{1}, \ldots, \alpha_{N}>0$ if

$$
\omega\left(f ; \delta_{1}, \ldots, \delta_{N}\right)=O\left(\prod_{j=1}^{N} \delta_{j}^{\alpha_{j}}\right), \quad \delta_{1}, \ldots, \delta_{N}>0 .
$$

Clearly, if $\gamma_{m_{1}, \ldots, m_{N}} \equiv 1, r \in(0,2)$, and $f \in \operatorname{Lip}\left(\alpha_{1}, \ldots, \alpha_{N}\right)$ for some

$$
\alpha_{j}>(2-r) / 2 r, \quad j=1,2, \ldots, N
$$

then $\sum(\gamma ; f)_{r}<\infty$.

Let $s \in \mathbb{R}_{+}$. Motivated by (1.32), a function $f=f\left(x_{1}, \ldots, x_{N}\right)$ periodic in each variable with period $2 \pi$ is said to be of bounded s-variation (in the sense of Vitali), in symbols: $f \in B V_{s}\left(\overline{\mathbb{T}}^{N}\right)$, if

$$
V_{s}(f):=
$$

$$
\sup \sum_{r_{1}=1}^{s_{1}} \ldots \sum_{r_{N}=1}^{s_{N}}\left|\Delta_{1, \ldots, 1}\left(f ; x_{1}^{r_{1}-1}, \ldots, x_{N}^{r_{N}-1} ; h_{1}^{r_{1}}, \ldots, h_{N}^{r_{N}}\right)\right|^{s}<\infty,
$$

where the supremum is extended over all partitions $\mathcal{P}:=\mathcal{P}_{1} \times \ldots \times \mathcal{P}_{N}$ of the closed cube $\overline{\mathbb{T}}^{N}$, where

$$
\mathcal{P}_{j}:-\pi=x_{j}^{0}<x_{j}^{1}<\cdots<x_{j}^{s_{j}}=\pi, s_{j} \geq 1, j=1,2, \ldots, N
$$


the operator $\Delta_{1}$ is defined in (1.53), and

$$
h_{j}^{r_{j}}:=x_{j}^{r_{j}}-x_{j}^{r_{j}-1} ; r_{j}=1,2, \ldots, s_{j} ; j=1,2, \ldots, N .
$$

Now, the extensions of Theorem 1.5 and Corollary 1.5 read as follows.

Theorem 1.8. Suppose $f \in C\left(\mathbb{T}^{N}\right) \cap B V_{s}\left(\overline{\mathbb{T}}^{N}\right)$ for some $s \in(0,2)$. If

$$
\gamma=\left\{\gamma_{m_{1}, \ldots, m_{N}}\right\} \in \mathcal{A}_{2 /(2-r)} \quad \text { for some } r \in(0,2),
$$

then

$$
\begin{gathered}
\sum(\gamma ; f)_{r} \leq \kappa C V_{s}^{r / 2}(f) \sum_{\mu_{1}=0}^{\infty} \ldots \sum_{\mu_{N}=0}^{\infty} 2^{-\left(\mu_{1}+\cdots+\mu_{N}\right) r} \times \\
\times \Gamma_{\mu_{1}-1, \ldots, \mu_{N}-1} \omega^{(2-s) r / 2}\left(f ; \frac{\pi}{2^{\mu_{1}}}, \ldots, \frac{\pi}{2^{\mu_{N}}}\right),
\end{gathered}
$$

where $\kappa$ is from 1.54 corresponding to $\alpha:=2 /(2-r)$; while $\sum(\gamma ; f)_{r}, \gamma_{\mu_{1}, \ldots, \mu_{N}}$, and $V_{s}(f)$ are defined in (1.55), (1.56), and (1.57), respectively.

Corollary 1.13. Under the conditions of Theorem 1.8, we have

$$
\begin{gathered}
\sum(\gamma ; f)_{r} \leq \kappa C V_{s}^{r / 2}(f) \sum_{m_{1}=1}^{\infty} \cdots \sum_{m_{N}=1}^{\infty}\left(\prod_{j=1}^{N} m_{j}^{-r}\right) \times \\
\times \gamma_{m_{1}, \ldots, m_{N}} \omega^{(2-s) r / 2}\left(f ; \frac{\pi}{m_{1}}, \ldots, \frac{\pi}{m_{N}}\right) .
\end{gathered}
$$

It follows that if $\gamma_{m_{1}, \ldots, m_{N}} \equiv 1, r \in(0,2), s \in(0,2)$, and $f \in \operatorname{Lip}\left(\alpha_{1}, \ldots, \alpha_{N}\right)$ with $\alpha_{j}>\max \{0,2(1-r) / r(2-s)\}, j=1,2, \ldots, N$, then $\sum(\gamma ; f)_{r}<\infty$. 


\section{Chapter 2}

\section{Walsh-Fourier series}

\subsection{Background: the Walsh-Paley system}

We consider the Walsh orthonormal system $\left\{w_{m}(x): m \in \mathbb{N}\right\}$ defined on the unit interval $\mathbb{I}:=[0,1)$ in the Paley enumeration, where $\mathbb{N}:=\{0,1,2, \ldots\}$. To go into some details, let

$$
r_{0}(x):=\left\{\begin{aligned}
1 & \text { if } x \in\left[0, \frac{1}{2}\right), \\
-1 & \text { if } x \in\left[\frac{1}{2}, 1\right)
\end{aligned}\right.
$$

and extend $r_{0}(x)$ for the half-axis $\mathbb{R}_{+}:=[0, \infty)$ with period 1 . The Rademacher orthonormal system $\left\{r_{k}(x): k \in \mathbb{N}\right\}$ is defined by

$$
r_{k}(x):=r_{0}\left(2^{k} x\right), \quad k=1,2, \ldots ; x \in \mathbb{I} .
$$

Now, the $m$ th Walsh function $w_{m}(x)$ in the Paley enumeration is defined as follows: If

$$
m=\sum_{k=0}^{\infty} m_{k} 2^{k}, \quad \text { each } m_{k}=0 \text { or } 1,
$$

is the binary decomposition of $m \in \mathbb{N}$, then set

$$
w_{m}(x):=\prod_{k=0}^{\infty} r_{k}^{m_{k}}(x), \quad x \in \mathbb{I} .
$$

Clearly, $m_{k}=0$ except for a finite number of $k$ 's. Thus, the right-hand side in (2.1) is a finite product for each $m \in \mathbb{N}$. In particular, we have

$$
w_{0}(x) \equiv 1 \quad \text { and } \quad w_{2^{m}}=r_{m}(x), \quad m \in \mathbb{N} .
$$


It is well known that $\left\{w_{m}(x): m \in \mathbb{N}\right\}$ is a complete orthonormal system on $\mathbb{I}$.

Any $x \in \mathbb{I}$ can be written in the form

$$
x=\sum_{k=0}^{\infty} x_{k} 2^{-k-1}, \quad \text { each } x_{k}=0 \text { or } 1 .
$$

For each $x \in \mathbb{I} \backslash Q$, there is only one expression of this form, where $Q$ is the collection of dyadic rationals in $\mathbb{I}$. When $x \in Q$ there are two expressions of this form, one which terminates in 0's and one which terminates in 1's. Now, the dyadic sum of $x, y \in \mathbb{I}$ is defined by

$$
x+y:=\sum_{k=1}^{\infty}\left|x_{k}-y_{k}\right| 2^{-k-1} .
$$

A remarkable property of the Walsh functions is that for each $m \in \mathbb{N}$, we have

$$
w_{m}\left(x_{1} \dot{+} x_{2}\right)=w_{m}\left(x_{1}\right) w_{m}\left(x_{2}\right), \quad x, y \in \mathbb{I}, \quad x \dot{+} y \notin Q .
$$

We recall that the dyadic topology of $\mathbb{I}$ is generated by the dyadic intervals

$$
I(k, m):=\left[k 2^{-m},(k+1) 2^{-m}\right), \quad 0 \leq k<2^{m} \quad \text { and } \quad k, m \in \mathbb{N} .
$$

A function $f: \mathbb{I} \rightarrow \mathbb{R}:=(-\infty, \infty)$ which is continuous from the dyadic topology to the usual topology is called $W$-continuous. Clearly, every classically continuous function on $\mathbb{I}$ is $W$-continuous, but the converse statement is not true. For example, every Walsh function $w_{m}(x)$ is $W$-continuous. The interested reader may consult the monograph [10, Ch.1, see especially pp. 8-15].

\subsection{Known results on single Walsh-Fourier series}

We briefly summarize the latest results related to the absolute convergence of single Walsh-Fourier series obtained in [5] by F. Móricz. 
Given a function $f: \mathbb{I} \rightarrow \mathbb{R}$, integrable in Lebesgue's sense on $\mathbb{I}=[0,1)$, its Walsh-Fourier series is defined by

$$
f(x) \sim \sum_{m \in \mathbb{N}} \hat{f}(m) w_{m}(x)
$$

where

$$
\hat{f}(m):=\int_{0}^{1} f(x, y) w_{m}(x) d x, \quad m \in \mathbb{N},
$$

are the Walsh-Fourier coefficients of $f$.

Denote by $C_{W}(\mathbb{I})$ the collection of $W$-continuous functions on $\mathbb{I}$.

The (global) dyadic modulus of continuity $\omega(f, \delta)$ of a function $f \in C_{W}$ is defined by

$$
\omega(f, \delta):=\{|f(x+y)-f(x)|: x \in[0,1), 0 \leq y<\delta\}
$$

and the dyadic $L^{p}$-modulus of continuity $\omega(f, \delta)_{p}$ of an $f \in L^{p}[0,1)$ is defined by

$$
\omega(f, \delta)_{p}:=\sup \left\{\left(\int_{0}^{1}|f(x+y)-f(x)|^{p}\right)^{1 / p}: 0 \leq y<\delta\right\} .
$$

We note that for each $n \in \mathbb{N}$, the condition $\delta<2^{-n}$ implies $\omega\left(w_{n}, \delta\right)=0$. Thus, the inequality

$$
\omega(f, 2 \delta) \leq 2 \omega(f, \delta), \quad \delta>0,
$$

does not hold for all $f \in C_{W}$. This is a sharp difference in comparison with the classical modulus of continuity of continuous functions.

For $\alpha>0$, we denote by $\operatorname{Lip}(\alpha, W)$ the collection of functions $f$ which satisfy

$$
\omega(f, \delta) \leq C \delta^{\alpha}, \quad 0<\delta<1
$$

where $C$ is a constant which depends only on $f$. Analogously, for $\alpha>0$ and $1 \leq p<\infty$, we denote by $\operatorname{Lip}\left(\alpha, L^{p}\right)$ the collection of functions $f \in L^{p}[0,1)$ 
which satisfy

$$
\omega(f, \delta)_{p} \leq C \delta^{\alpha}, \quad 0<\delta<1
$$

Clearly, if $f \in \operatorname{Lip}(\alpha, W)$ for some $\alpha>0$, then $f \in C_{W}$; furthermore, for any $\alpha>0$ and $1 \leq p<\infty$, we have

$$
\operatorname{Lip}(\alpha, W) \subset \operatorname{Lip}(\alpha, W)_{p}
$$

We note that the contrast to the classical Lipschitz classes, the dyadic Lipschitz classes $\operatorname{Lip}(\alpha, W)$ and $\operatorname{Lip}(\alpha, W)_{p}$ are not trivial for $\alpha>1$.

For each dyadic interval $I$ and each $f \in C_{W}$, the local dyadic modulus of continuity $\omega(f, I)$ is defined by

$$
\omega(f, I):=\{|f(x+y)-f(x)|: x \in I, 0 \leq y<|I|\},
$$

where by $|I|$ we denote the length of the interval $I$. Moreover, for each dyadic interval $I$ and each $f \in L^{p}, 1 \leq p<\infty$, the local dyadic $L^{p}$-modulus of continuity is defined by

$$
\omega(f, I)_{p}:=\sup \left\{\left(\frac{1}{|I|} \int_{I}|f(x+y)-f(x)|^{p}\right)^{1 / p}: 0 \leq y<|I|\right\} .
$$

Roughly speaking, the dyadic local modulus of continuity is a measure of the oscillation of $f$ on the dyadic interval $I$. Thus, we say that a function $\mathrm{f}$ is of s-bounded fluctuation for some $0<s \infty$ on the whole interval $[0,1)$, in symbols: $f \in B F_{s}$, if

$$
\mathcal{F} l_{s}(f):=\sup _{n \geq 1}\left(\sum_{k=0}^{2^{n}-1}|\omega(f, I(k, n))|^{s}\right)^{1 / s}<\infty ;
$$

and $\mathcal{F} l_{s}(f)$ is called the total s-fluctuation of $f$ on $[0,1)$. It is easy to check that

$$
B F_{s_{1}} \subset B F_{s_{2}} \quad \text { if } \quad 0<s_{1}<s_{2}<\infty \text {. }
$$


We note that every function of bounded variation (in the classical sense) on the interval $[0,1]$ is also of s-bounded fluctuation for $s=1$, but the converse statement is false.

The first result is formulated in terms of the dyadic $L^{p}$-modulus of continuity.

Theorem 2.1. If $f \in L^{p}[0,1)$ for some $1<p \leq 2$ and

$$
\left\{\gamma_{m}\right\} \in \mathfrak{A}_{p /(p-r p+r)} \quad \text { for some } \quad 0<r<q
$$

where $1 / p+1 / q=1$, then

$$
\sum_{n=1}^{\infty} \gamma_{m}|\hat{f}(m)|^{r} \leq 2^{-r} \kappa \sum_{\mu=0}^{\infty} 2^{-\mu r / q} \Gamma_{\mu-1}\left|\omega\left(f, 2^{-\mu}\right)_{p}\right|^{r}
$$

where $\kappa$ is from (1.1) corresponding to $\alpha:=p /(p-r p+r), \Gamma_{\mu}$ is defined in (1.6) and $D_{\mu}$ is defined in (1.2).

The next result is formulated in terms of the local dyadic $L^{p}$-modulus of continuity.

Theorem 2.2. If $f \in L^{p}[0,1)$ for some $1<p \leq 2$ and $\gamma_{m} \subset \mathbb{R}_{+}$satisfies condition (2.5), where $1 / p+1 / q=1$, then

$$
\sum_{m=1}^{\infty} \gamma_{m}|\hat{f}(m)|^{r} \leq 2^{-r} \kappa \sum_{\mu=0}^{\infty} 2^{-\mu r} \Gamma_{\mu-1}\left(\sum_{k=0}^{2^{\mu}-1}\left|\omega(f, I(k, \mu))_{p}\right|^{p}\right)^{r / p},
$$

where $\kappa$ is from (1.1) corresponding to $\alpha:=p /(p-r p+r), \Gamma_{\mu}$ is defined in (1.6) and $I(k, \mu)$ is defined in (2.3).

The sufficient conditions above can be relaxed if $f$ is of bounded fluctuation.

Theorem 2.3. If $f \in C_{W} \cap B F_{s}$ for some $0<s<2$ and if

$$
\left\{\gamma_{m}\right\} \in \mathfrak{A}_{2 /(2-r)} \quad \text { for some } 0<r<2
$$


then

$$
\sum_{m=1}^{\infty} \gamma_{m}|\hat{f}(m)|^{r} \leq 2^{-r} \kappa\left|\mathcal{F} l_{s}(f)\right|^{r s / 2} \sum_{\mu=0}^{\infty} 2^{-\mu r} \Gamma_{\mu-1}\left|\omega\left(f, 2^{-\mu}\right)\right|^{(2-s) r / 2},
$$

where where $\kappa$ is from (1.1) corresponding to $\alpha:=2 /(2-r), \mathcal{F} l_{s}(f)$ is defined in (2.4) and $\Gamma_{\mu}$ is defined in (1.6)

\subsection{New results on double Walsh-Fourier series}

Given a function $f: \mathbb{I}^{2} \rightarrow \mathbb{R}$, integrable in Lebesgue's sense on the unit square $\mathbb{I}^{2}=[0,1) \times[0,1)$, in symbols: $f \in L^{1}\left(\mathbb{I}^{2}\right)$, its double Walsh-Fourier series is defined by

$$
f(x, y) \sim \sum_{m \in \mathbb{N}} \sum_{n \in \mathbb{N}} \hat{f}(m, n) w_{m}(x) w_{n}(y)
$$

where the

$$
\hat{f}(m, n):=\int_{0}^{1} \int_{0}^{1} f(x, y) w_{m}(x) w_{n}(y) d x d y, \quad m, n \in \mathbb{N}
$$

are the Walsh-Fourier coefficients of $f$.

Denote by $C_{W}\left(\mathbb{I}^{2}\right)$ the collection of $W$-continuous functions on $\mathbb{I}^{2}$, where the dyadic topology on $\mathbb{I}^{2}$ is generated by dyadic rectangles

$$
I(k, m ; l, n):=I(k, m) \times I(l, n)
$$

$=\left[k 2^{-m},(k+1) 2^{-m}\right) \times\left[l 2^{-n},(l+1) 2^{-n}\right), \quad 0 \leq k<2^{m}, 0 \leq l<2^{n}$ and $k, l, m, n \in \mathbb{N}$ (cf (2.3)). The (global) dyadic modulus of continuity of a function $f \in C_{W}\left(\mathbb{I}^{2}\right)$ is defined by

$$
\omega\left(f ; \delta_{1}, \delta_{2}\right):=\sup \left\{\left|\Delta_{1,1} f\left(x, y ; h_{1}, h_{2}\right)\right|:(x, y) \in \mathbb{I}^{2}\right.
$$




$$
\left.0 \leq h_{j}<\delta_{j}, j=1,2\right\}, \quad 0<\delta_{j} \leq 1
$$

We recall that the difference operators $\Delta_{1,0}, \Delta_{0,1}$ and $\Delta_{1,1}$ are defined in the usual way:

$$
\begin{aligned}
& \Delta_{1,0} f\left(x, y ; h_{1}\right):=f\left(x+h_{1}, y\right)-f(x, y), \\
& \Delta_{0,1} f\left(x, y ; h_{2}\right):=f\left(x, y \dot{+} h_{2}\right)-f(x, y),
\end{aligned}
$$

and $\Delta_{1,1}=\Delta_{1,0} \Delta_{0,1}=\Delta_{0,1} \Delta_{1,0}$, that is,

$$
\begin{gathered}
\Delta_{1,1} f\left(x, y ; h_{1}, h_{2}\right):=\mid f\left(x \dot{+} h_{1}, y \dot{+} h_{2}\right)-f\left(x, y \dot{+} h_{2}\right) \\
-f\left(x \dot{+} h_{1}, y\right)+f(x, y) .
\end{gathered}
$$

Furthermore, the dyadic $L^{p}$-modulus of continuity of a function $f \in L^{p}(\mathbb{I})$ for some $1 \leq p<\infty$ is defined by

$$
\begin{gathered}
\omega\left(f ; \delta_{1}, \delta_{2}\right)_{p}:=\sup \left\{\left(\int_{0}^{1} \int_{0}^{1}\left|\Delta_{1,1} f\left(x, y ; h_{1}, h_{2}\right)\right|^{p} d x d y\right)^{1 / p}:\right. \\
\left.0 \leq h_{j}<\delta_{j}, j=1,2\right\} .
\end{gathered}
$$

For $\alpha_{1}, \alpha_{2}>0$, the dyadic Lipschitz class $\operatorname{Lip}\left(\alpha_{1}, \alpha_{2} ; W\right)$ is the collection of those functions $f \in C_{W}\left(\mathbb{I}^{2}\right)$ which satisfy the inequality

$$
\omega\left(f ; \delta_{1}, \delta_{2}\right) \leq C \delta_{1}^{\alpha_{1}} \delta_{2}^{\alpha_{2}}, \quad 0<\delta_{1}, \delta_{2} \leq 1
$$

where $C$ is a constant which depends only on $f$. Analogously, for $\alpha_{1}, \alpha_{2}>0$ and $1 \leq p<\infty$, we denote by $\operatorname{Lip}\left(\alpha_{1}, \alpha_{2} ; L^{p}\right)$ the collection of functions $f \in L^{p}\left(\mathbb{I}^{2}\right)$ which satisfy the inequality

$$
\omega\left(f ; \delta_{1}, \delta_{2}\right)_{p} \leq C \delta_{1}^{\alpha_{1}} \delta_{2}^{\alpha_{2}}, \quad 0<\delta_{1}, \delta_{2} \leq 1
$$

For each dyadic rectangle

$$
I(k, m ; l, n):=I(k, m) \times I(l, n)=: I \times J,
$$


where $0 \leq k<2^{m}, 0 \leq l<2^{n} ; k, l, m, n \in \mathbb{N}$ (see (2.8)), the local dyadic modulus of continuity of a function $f \in C_{W}\left(\mathbb{I}^{2}\right)$ is defined by

$$
\begin{gathered}
\omega(f ; I \times J):=\sup \left\{\left|\Delta_{1,1} f\left(x, y ; h_{1}, h_{2}\right)\right|:(x, y) \in I \times J,\right. \\
\left.0 \leq h_{1}<|I|, 0 \leq h_{2}<|J|\right\},
\end{gathered}
$$

where $|I|=2^{-m}$ and $|J|=2^{-n}$ are the length of the intervals $I$ and $J$, respectively. Morever, for each $1 \leq p<\infty$, the local dyadic $L^{p}$-modulus of continuity of a function $f \in L^{p}\left(\mathbb{I}^{2}\right)$ is defined by

$$
\begin{gathered}
\omega\left(f ; \delta_{1}, \delta_{2}\right)_{p}:=\sup \left\{\left(\frac{1}{|I| \cdot|J|} \int_{I} \int_{J}\left|\Delta_{1,1} f\left(x, y ; h_{1}, h_{2}\right)\right|^{p} d x d y\right)^{1 / p}:\right. \\
\left.0 \leq h_{1}<|I|, 0 \leq h_{2}<|J|\right\} .
\end{gathered}
$$

Finally, we say that a function $f: \mathbb{I}^{2} \rightarrow \mathbb{R}$ is of s-bounded fluctuation for some $0<s<\infty$, in symbols: $f \in B F_{s}\left(\mathbb{I}^{2}\right)$, if

$$
F l_{s}\left(f ; \mathbb{I}^{2}\right):=\sup _{m \geq 1} \sup _{n \geq 1}\left(\sum_{k=0}^{2^{m}-1} \sum_{l=0}^{2^{n}-1}|\omega(f ; I(k, m) \times I(l, n))|^{s}\right)^{1 / s}<\infty
$$

and $F l_{s}\left(f ; \mathbb{I}^{2}\right)$ may be called the total s-fluctuation of $f$ over $\mathbb{I}^{2}$. It is easy to check that

$$
B F_{s_{1}} \subset B F_{s_{2}}, \quad 0<s_{1}<s_{2}<\infty .
$$

Extend all the Walsh functions $w_{m}(x)$ for $\mathbb{R}_{+}$with period 1 ; in particular, set

$$
w_{m}(1):=w_{m}(0), \quad m \in \mathbb{N} .
$$

It is worth noting that every function of bounded variation in the sense of Vitali (see, e.g., [3]) on the closed unit square $[0,1] \times[0,1]$ is also of $s$-bounded fluctuation on $\mathbb{I}^{2}$ for $s=1$. The converse statement is false. There exists a function $f \in B F_{1}\left(\mathbb{I}^{2}\right)$ such that

$$
f(x, 1)=f(x, 0), \quad f(1, y)=f(0, y) \quad \text { for all } \quad 0 \leq x, y \leq 1
$$


(all the Walsh functions $w_{m}(x) w_{n}(y)$ are such ones), and $f$ is not of bounded variation in the sense of Vitali.

Let $f: \mathbb{I}^{2} \rightarrow \mathbb{R}$ be a function either in $C_{W}\left(\mathbb{I}^{2}\right)$ or in $L^{p}\left(\mathbb{I}^{2}\right)$ for some $1<p \leq 2$. Our aim is to give best possible sufficient conditions for the finiteness of the double series

$$
\sum_{m=1}^{\infty} \sum_{n=1}^{\infty} \gamma_{m n}|\hat{f}(m, n)|^{r}
$$

where $\left\{\gamma_{m n}\right\}$ is a double sequence of nonnegative real numbers satisfying a mild assumption and $0<r<2$. The restriction $r<2$ is explained by the fact that if $f \in C_{W}\left(\mathbb{I}^{2}\right)$ or only $f \in L^{2}\left(\mathbb{I}^{2}\right)$, then by the Parseval formula we have

$$
\sum_{m=0}^{\infty} \sum_{n=0}^{\infty}|\hat{f}(m, n)|^{2}=\int_{0}^{1} \int_{0}^{1}|f(x, y)|^{2} d x d y<\infty .
$$

Our first result in this section is formulated in terms of the dyadic $L^{p_{-}}$ modulus of continuity.

Theorem 2.4. Suppose $f \in L^{p}\left(\mathbb{I}^{2}\right)$ for some $1<p \leq 2$. If

$$
\left\{\gamma_{m n}\right\} \in \mathfrak{A}_{p /(p-r p+r)} \text { for some } 0<r<q, \text { where } \frac{1}{p}+\frac{1}{q}=1,
$$

then

$$
\begin{gathered}
\sum_{m=1}^{\infty} \sum_{n=1}^{\infty} \gamma_{m n}|\hat{f}(m, n)|^{r} \\
\leq 4^{-r} \kappa \sum_{\mu=0}^{\infty} \sum_{\nu=0}^{\infty} 2^{-(\mu+\nu) r / q} \Gamma_{\mu-1, \nu-1}\left|\omega\left(f ; 2^{-\mu}, 2^{-\nu}\right)_{p}\right|^{r},
\end{gathered}
$$

where $\kappa$ is from (1.9) correspondings to $\alpha:=p /(p-r p+r)$,

$$
\Gamma_{\mu \nu}:=\sum_{m \in D_{\mu}} \sum_{n \in D_{\nu}} \gamma_{m n}, \quad \mu, \nu \in \mathbb{N}
$$

and $D_{\mu}$ is defined in (1.2). 
Proof. Fix $\mu, \nu \in \mathbb{N}$ and $\left(h_{1}, h_{2}\right) \in I(1, \mu+1 ; 1, \nu+1)$ (see (2.8)). Set

$$
g(x, y):=\Delta_{1,1} f\left(x, y ; h_{1}, h_{2}\right), \quad(x, y) \in \mathbb{I}^{2}
$$

(see (2.9)). It follows from (2.1) that

$$
w_{m}\left(h_{1}\right)=-1 \quad \text { if } \quad m \in D_{\mu} \quad \text { and } \quad h_{1} \in I(1, \mu+1):=\left[2^{-\mu-1}, 2^{-\mu}\right),
$$

and

$$
w_{n}\left(h_{2}\right)=-1 \quad \text { if } \quad n \in D_{\nu} \quad \text { and } \quad h_{2} \in I(1, \nu+1):=\left[2^{-\nu-1}, 2^{-\nu}\right) .
$$

By (2.2), we find that

$$
\begin{gathered}
\hat{g}(m, n):=\int_{0}^{1} \int_{0}^{1} g(x, y) w_{m}(x) w_{n}(y) d x d y \\
=\int_{0}^{1} \int_{0}^{1} f(x, y)\left\{w_{m}\left(x \dot{+} h_{1}\right) w_{n}\left(y \dot{+} h_{2}\right)-w_{m}(x) w_{n}\left(y \dot{+} h_{2}\right)\right. \\
\left.-w_{m}\left(x \dot{+} h_{1}\right) w_{n}(y)+w_{m}(x) w_{n}(y)\right\} d x d y \\
=\left\{w_{m}\left(h_{1}\right) w_{n}\left(h_{2}\right)-w_{n}\left(h_{2}\right)-w_{m}\left(h_{1}\right)+1\right\} \hat{f}(m, n)=4 \hat{f}(m, n) .
\end{gathered}
$$

By virtue of the Hausdorff-Young inequality (see, e.g., [19, Vol. II, pp. 101-103] in the case of single orthogonal series, but the scheme of its proof easily applies for multiple orthogonal series; see also [11, p. 178], where this inequality is formulated in terms of multiple Fourier integrals), for any $1<p \leq 2$ we obtain that

$$
\begin{gathered}
\left(\sum_{m \in D_{\mu}} \sum_{n \in D_{\nu}}|\hat{f}(m, n)|^{q}\right)^{1 / q}=\frac{1}{4}\left(\sum_{m \in D_{\mu}} \sum_{n \in D_{\nu}}|\hat{g}(m, n)|^{q}\right)^{1 / q} \\
\leq \frac{1}{4}\left(\int_{0}^{1} \int_{0}^{1}\left|\Delta_{1,1} f\left(x, y ; h_{1}, h_{2}\right)\right|^{p} d x d y\right)^{1 / p}
\end{gathered}
$$




$$
\leq \frac{1}{4} \omega\left(f ; 2^{-\mu}, 2^{-\nu}\right)_{p}, \quad \mu, \nu \in \mathbb{N} \quad \text { and } \quad \frac{1}{p}+\frac{1}{q}=1 .
$$

Applying Hölder's inequality with the exponents

$$
\frac{q}{r}=\frac{p}{r(p-1)} \text { and } \frac{q}{q-r}=\frac{p}{p-r p+r}
$$

it follows from (1.9), (2.12) and (2.17) that

$$
\begin{gathered}
\sum_{m \in D_{\mu}} \sum_{n \in D_{\nu}} \gamma_{m n}|\hat{f}(m, n)|^{r} \\
\leq\left(\sum_{m \in D_{\mu}} \sum_{n \in D_{\nu}}|\hat{f}(m, n)|^{q}\right)^{r / q}\left(\sum_{m \in D_{\mu}} \sum_{n \in D_{\nu}} \gamma_{m n}^{p /(p-r p+r)}\right)^{(p-r p+r) / p} \\
\leq \kappa 4^{-r} 2^{-(\mu+\nu) r / q} \Gamma_{\mu-1, \nu-1}\left|\omega\left(f ; 2^{-\mu}, 2^{-\nu}\right)_{p}\right|^{r}, \quad \mu, \nu \in \mathbb{N}
\end{gathered}
$$

where $\Gamma_{\mu \nu}$ is defined in (2.14).

Summing inequality (2.19) over $\mu, \nu \in \mathbb{N}$ yields (2.13) to be proved.

It is worth formulating Theorem 2.4 in the particular case when $f \in$ $\operatorname{Lip}\left(\alpha_{1}, \alpha_{2} ; W\right)_{p}$ and $\gamma_{m n} \equiv 1$.

Corollary 2.1. Suppose $f \in \operatorname{Lip}\left(\alpha_{1}, \alpha_{2} ; W\right)_{p}$ for some $\alpha_{1}, \alpha_{2}>0$ and $1<$ $p \leq 2$. If

$$
\frac{q}{1+q \min \left\{\alpha_{1}, \alpha_{2}\right\}}<r<q
$$

then

$$
\sum_{m=1}^{\infty} \sum_{n=1}^{\infty}|\hat{f}(m, n)|^{r}<\infty
$$

We note that Corollary 2.1 for $p=q=2$ is an extension of [10, Theorem 10 on p. 67] from single to double Walsh-Fourier series. In case $p=q=2$ 
and $r=1$, condition (2.20) is satisfied whenever $\min \left\{\alpha_{1}, \alpha_{2}\right\}>1 / 2$. On the other hand, there exists a function $g: \mathbb{I} \rightarrow \mathbb{R}$ such that

$$
g \in \operatorname{Lip}(1 / 2, W) \quad \text { and } \quad \sum_{m=1}^{\infty}|\hat{g}(m)|=\infty
$$

(see [10, p. 68]). Now, define

$$
f(x, y):=g(x) g(y), \quad(x, y) \in \mathbb{I}^{2},
$$

then $f \in \operatorname{Lip}\left(\alpha_{1}, \alpha_{2} ; W\right)$ and condition (2.21) is not satisfied.

It is of interest to observe that if a function $f$ belongs to the narrower class $\operatorname{Lip}\left(\alpha_{1}, \alpha_{2} ; W\right)$ instead of $\operatorname{Lip}\left(\alpha_{1}, \alpha_{2} ; W\right)_{p}$, then the restriction $r<q$ in (2.20) is superfluous. This is due to the fact that $\operatorname{Lip}\left(\alpha_{1}, \alpha_{2} ; W\right) \subset L^{2}\left(\mathbb{I}^{2}\right)$, and by the Parseval formula, we have

$$
\{\hat{f}(m, n): m, n \geq 0\} \in l^{2} \subset l^{r} \quad \text { for all } \quad r \geq 2 \text {. }
$$

Next, we formulate Theorem 2.4 in the particular case when $\gamma_{m n}=$ $m^{\beta_{1}} n^{\beta_{2}}$ and $r=1$.

Corollary 2.2. Suppose $f \in \operatorname{Lip}\left(\alpha_{1}, \alpha_{2} ; W\right)_{p}$ for some $\alpha_{1}, \alpha_{2}>0$ and $1<$ $p \leq 2$. If $\beta_{1}, \beta_{2} \in \mathbb{R}$ are such that

$$
\beta_{j}<\alpha_{j}-\frac{1}{p}, \quad j=1,2
$$

then

$$
\sum_{m=1}^{\infty} \sum_{n=1}^{\infty} m^{\beta_{1}} n^{\beta_{2}}|\hat{f}(m, n)|<\infty
$$

We note that Corollary 2.2 for $p=2$ is the extension of [10, Theorem 11 on p. 68 formulated with $(-\beta)$ in place of $\beta$ ] from single to double WalshFourier series. We also note that it is the dyadic analogue of the theorem of 
was O. Szász proved for single trigonometric series (see, e.g., [19, Vol. I, pp. 243]).

Our second new result in this section is formulated in terms of the local dyadic $L^{p}$-modulus of continuity.

Theorem 2.5. Suppose $f \in L^{p}\left(\mathbb{I}^{2}\right)$ for some $1<p \leq 2$. If $\left\{\gamma_{m n} \geq 0\right\}$ satisfies condition (2.12), then

$$
\sum_{m=1}^{\infty} \sum_{n=1}^{\infty} \gamma_{m n}|\hat{f}(m, n)|^{r}
$$

$$
\leq 4^{-r} \kappa \sum_{\mu=0}^{\infty} \sum_{\nu=0}^{\infty} 2^{-(\mu+\nu) r} \Gamma_{\mu-1, \nu-1}\left(\sum_{k=0}^{2^{\mu}-1} \sum_{l=0}^{2^{\nu}-1}\left|\omega(f ; I(k, \mu ; l, \nu))_{p}\right|^{p}\right)^{1 / p}
$$

where $\kappa$ is from (1.9) corresponding to $\alpha:=p /(p-r p+r), \Gamma_{\mu-1, \nu-1}$ is defined in $(2.14)$, and $I(k, \mu ; l, \nu)$ is defined in (2.8).

Proof. Let $\mu, \nu \in \mathbb{N}$ and $\left(h_{1}, h_{2}\right) \in I(1, \mu+1 ; 1, \nu+1)$. Proceeding as in the proof of Theorem 1 (see (2.17)), we obtain that

$$
\begin{gathered}
\left(\sum_{m \in D_{\mu}} \sum_{n \in D_{\nu}}|\hat{f}(m, n)|^{q}\right)^{1 / q} \\
\leq \frac{1}{4}\left(\int_{0}^{1} \int_{0}^{1}\left|\Delta_{1,1} f\left(x, y ; h_{1}, h_{2}\right)\right|^{p} d x d y\right)^{1 / p} \\
=\frac{1}{4}\left(\sum_{k=0}^{2^{\mu}-1} \sum_{l=0}^{2^{\nu}-1} \int_{I(k, \mu)} \int_{I(l, \nu)}\left|\Delta_{1,1} f\left(x, y ; h_{1}, h_{2}\right)\right|^{p} d x d y\right)^{1 / p} \\
\leq \frac{1}{4}\left(\sum_{k=0}^{2^{\mu}-1} \sum_{l=0}^{2^{\nu}-1} 2^{-(\mu+\nu)}\left|\omega(f ; I(k, \mu ; l, \nu))_{p}\right|^{p}\right)^{1 / p} .
\end{gathered}
$$

Applying Hölder's inequality with the exponents (2.18), it follows from (1.9), (2.12) and (2.25) that

$$
\sum_{m \in D_{\mu}} \sum_{n \in D_{\nu}} \gamma_{m n}|\hat{f}(m, n)|^{r}
$$




$$
\begin{gathered}
\leq\left(\sum_{m \in D_{\mu}} \sum_{n \in D_{\nu}}|\hat{f}(m, n)|^{r}\right)^{r / q}\left(\sum_{m \in D_{\mu}} \sum_{n \in D_{\nu}} \gamma_{m n}^{p /(p-r p+r)}\right)^{(p-r p+r) / p} \\
\leq \kappa 4^{-r} 2^{-(\mu+\nu) r / q} \Gamma_{\mu-1, \nu-1}\left(\sum_{k=0}^{2^{\mu}-1} \sum_{l=0}^{2^{\nu}-1} 2^{-(\mu+\nu)}\left|\omega(f ; I(k, \mu ; l, \nu))_{p}\right|^{p}\right)^{r / p} \\
=\kappa 4^{-r} 2^{-(\mu+\nu) r} \Gamma_{\mu-1, \nu-1}\left(\sum_{k=0}^{2^{\mu}-1} \sum_{l=0}^{2^{\nu}-1}\left|\omega(f ; I(k, \mu ; l, \nu))_{p}\right|^{p}\right)^{r / p} .
\end{gathered}
$$

Summing inequality (2.26) over $\mu, \nu \in \mathbb{N}$ yields (2.24) to be proved.

We note that Corollaries 2.1 and 2.2 can also be deduced from Theorem 2.5. Furthermore, the special case when $\gamma_{m n} \equiv 1$ and $r=1$, Theorem 2.2 is an extension of [10, Theorem 9 on p. 64 formulated with $\omega(f, I(k, \mu))$ in place of $\left.\omega(f, I(k, \mu))_{p}\right]$ from single to double Walsh-Fourier series.

The sufficient conditions above can be relaxed if the function $f: \mathbb{I}^{2} \rightarrow \mathbb{R}$ is of bounded fluctuation. Our third new result is the following

Theorem 2.6. Suppose $f \in C_{W} \cap B F_{s}\left(\mathbb{I}^{2}\right)$ for some $0<s<2$. If

$$
\left\{a_{m n} \geq 0\right\} \in \mathfrak{A}_{2 /(2-r)} \text { for some } 0<r<2,
$$

then

$$
\begin{gathered}
\sum_{m=1}^{\infty} \sum_{n=1}^{\infty} \gamma_{m n}|\hat{f}(m, n)|^{r} \\
\leq 4^{-r} \kappa\left|F l_{s}\left(f ; \mathbb{I}^{2}\right)\right|^{r s / 2} \sum_{\mu=0}^{\infty} \sum_{\nu=0}^{\infty} 2^{-(\mu+\nu) r} \Gamma_{\mu-1, \nu-1}\left|\omega\left(f ; 2^{-\mu}, 2^{-\nu}\right)\right|^{(1-s / 2) r},
\end{gathered}
$$

where $\kappa$ is from (1.9) corresponding to $\alpha:=2 /(2-r), F l_{s}(f)$ is defined in (2.10) and $\Gamma_{\mu \nu}$ is defined in (2.14).

Proof. Let $\mu, \nu \in \mathbb{N}$ and $\left(h_{1}, h_{2}\right) \in I(1, \mu+1 ; 1, \nu+1)$. Making use of $(2.15)$ and (2.16), this time the Parseval formula $(r=2)$ gives

$$
\left(\sum_{m \in D_{\mu}} \sum_{n \in D_{\nu}}|\hat{f}(m, n)|^{2}\right)^{1 / 2}=\frac{1}{4}\left(\sum_{m \in D_{\mu}} \sum_{n \in D_{\nu}}|\hat{g}(m, n)|^{2}\right)^{1 / 2}
$$




$$
\begin{gathered}
\leq \frac{1}{4}\left(\int_{0}^{1} \int_{0}^{1}|g(x, y)|^{2} d x d y\right)^{1 / 2}=\frac{1}{4}\left(\int_{0}^{1} \int_{0}^{1}\left|\Delta_{1,1} f\left(x, y ; h_{1}, h_{2}\right)\right|^{2} d x d y\right)^{1 / 2} \\
=\frac{1}{4}\left(\sum_{k=0}^{2^{\mu}-1} \sum_{l=0}^{2^{\nu}-1} \int_{I(k, \mu)} \int_{I(l, \nu)}\left|\Delta_{1,1} f\left(x, y ; h_{1}, h_{2}\right)\right|^{2} d x d y\right)^{1 / 2} \\
\leq \frac{1}{4}\left(\sum_{k=0}^{2^{\mu}-1} \sum_{l=0}^{2^{\nu}-1} 2^{-(\mu+\nu)}|\omega(f ; I(k, \mu ; l, \nu))|^{2}\right)^{1 / 2} .
\end{gathered}
$$

Next, applying Hölder's inequality with the exponents $2 / r$ and $2 /(2-r)$, it follows from (1.9), (2.27) and (2.29) that

$$
\begin{gathered}
\sum_{m \in D_{\mu}} \sum_{n \in D_{\nu}} \gamma_{m n}|\hat{f}(m, n)|^{r} \\
\leq\left(\sum_{m \in D_{\mu}} \sum_{n \in D_{\nu}}|\hat{f}(m, n)|^{2}\right)^{r / 2}\left(\sum_{m \in D_{\mu}} \sum_{n \in D_{\nu}} \gamma_{m n}^{2 /(2-r)}\right)^{(2-r) / r} \\
\leq \kappa 4^{-r} 2^{-(\mu+\nu) r / 2} \Gamma_{\mu-1, \nu-1}\left(\sum_{m \in D_{\mu}} \sum_{n \in D_{\nu}}|\hat{f}(m, n)|^{2}\right)^{r / 2} .
\end{gathered}
$$

Combining (2.29) and (2.30), we conclude that

$$
\begin{gathered}
\sum_{m \in D_{\mu}} \sum_{n \in D_{\nu}} \gamma_{m n}|\hat{f}(m, n)|^{r} \leq \kappa 4^{-r} 2^{-(\mu+\nu) r / 2} \times \\
\times \Gamma_{\mu-1, \nu-1}\left(2^{-(\mu+\nu)} \sum_{k=0}^{2^{\mu}-1} \sum_{l=0}^{2^{\nu}-1}|\omega(f ; I(k, \mu ; l, \nu))|^{2}\right)^{r / 2} \\
=\kappa 4^{-r} 2^{-(\mu+\nu) r} \Gamma_{\mu-1, \nu-1} \times \\
\left(\sum_{k=0}^{2^{\mu}-1} \sum_{l=0}^{2^{\nu}-1}|\omega(f ; I(k, \mu ; l, \nu))|^{2-s}|\omega(f ; I(k, \mu ; l, \nu))|^{s}\right)^{r / 2} \\
\leq \kappa 4^{-r} 2^{-(\mu+\nu) r} \Gamma_{\mu-1, \nu-1}\left|\omega\left(f ; 2^{-\mu}, 2^{-\nu}\right)\right|^{(2-s) r / 2}\left|F l_{s}\left(\mathbb{I}^{2}\right)\right|^{r s / 2} .
\end{gathered}
$$

Summing inequality (2.31) over $\mu, \nu \in \mathbb{N}$ yields (2.28) to be proved.

We formulate Theorem 2.6 in the particular case when the function $f \in$ $B F_{s}\left(\mathbb{I}^{2}\right)$ belongs to a dyadic Lipschitz class and $\gamma_{m n} \equiv 1$. 
Corollary 2.3. Suppose $f \in \operatorname{Lip}\left(\alpha_{1}, \alpha_{2} ; W\right) \cap B F_{s}\left(\mathbb{I}^{2}\right)$ for some $\alpha_{1}, \alpha_{2}>0$ and $0<s<2$. If

$$
r>\frac{1}{1+(1-s / 2) \min \left\{\alpha_{1}, \alpha_{2}\right\}},
$$

then (2.21) is satisfied.

We note that Corollary 2.3 is an extension of [10, Corollary 4 on p. 67 formulated for $r=1$ and $1 \leq s<2$ ] from single to double Walsh-Fourier series

Finally, we formulate Theorem 2.6 in the special case when $r=1$ and $\gamma_{m n}=m^{\beta_{1}} n^{\beta_{2}}$, where $\beta_{1}, \beta_{2} \in \mathbb{R}$.

Corollary 2.4. Suppose $f \in \operatorname{Lip}\left(\alpha_{1}, \alpha_{2} ; W\right) \cap B F_{s}\left(\mathbb{I}^{2}\right)$ for some $\alpha_{1}, \alpha_{2}>0$ and $0<s<2$. If

$$
\beta_{j}<(1-s / 2) \alpha_{j}, \quad j=1,2,
$$

then (2.23) is satisfied.

\subsection{Absolute convergence of double Walsh-Fourier series}

Denote by $\mathcal{A}\left(\mathbb{I}^{2}\right)$ the collection of functions $f \in L^{1}\left(\mathbb{I}^{2}\right)$ whose double Walsh-Fourier series converge absolutely. Since

$$
\left|w_{m}(x)\right|=\left|w_{n}(y)\right|=1 \quad \text { for all } \quad m, n \in \mathbb{N} \quad \text { and } \quad(x, y) \in \mathbb{I}^{2}
$$

it is clear that $f \in \mathcal{A}\left(\mathbb{I}^{2}\right)$ if and only if

$$
\|f\|_{\mathcal{A}\left(\mathbb{I}^{2}\right)}:=\sum_{m=0}^{\infty} \sum_{n=0}^{\infty}|\hat{f}(m, n)|<\infty .
$$


Morever, each $f \in \mathcal{A}\left(\mathbb{I}^{2}\right)$ is a $W$-continuous function, since it has a uniformly convergent Walsh-Fourier series.

We note that $\|\cdot\|_{\mathcal{A}\left(\mathbb{I}^{2}\right)}$ is a norm. Since the mapping $f \rightarrow\{\hat{f}(m, n)$ : $m, n \in \mathbb{N}\}$ is a one-to-one linear isometry from $\mathcal{A}\left(\mathbb{I}^{2}\right)$ to $l^{1}$, it is evident that $\mathcal{A}\left(\mathbb{I}^{2}\right)$ is a Banach space. Similarly to the case of single Walsh-Fourier series, $\mathcal{A}\left(\mathbb{I}^{2}\right)$ is even a Banach algebra with respect to pointwise multiplication (see details in [10, pp. 63-64]).

If a function $f \in L^{1}\left(\mathbb{I}^{2}\right)$ is such that

$$
\hat{f}(m, n)=0 \quad \text { whenever } \min \{m, n\}=0,
$$

then each of the conditions (2.13), (2.24) and (2.28) in case $a_{m n} \equiv 1$ and $r=1$, condition (2.21) in case $r=1$, and condition (2.23) in case $\beta_{1}=\beta_{2}=0$ is sufficient to conclude (2.34).

If condition (2.35) is not satisfied, we may proceed as follows. Observe that

$$
\hat{f}(m, 0)=\hat{f}_{1}(m), \quad \text { where } \quad f_{1}(x):=\int_{0}^{1} f(x, y) d y, \quad x \in \mathbb{I}
$$

and

$$
\hat{f}(0, n)=\hat{f}_{2}(n), \quad \text { where } \quad f_{2}(y):=\int_{0}^{1} f(x, y) d x, \quad y \in \mathbb{I} .
$$

By Hölder's inequality, we have $f_{1}, f_{2} \in L^{p}(\mathbb{I})$ whenever $f \in L^{p}\left(\mathbb{I}^{2}\right)$ for some $1 \leq p<\infty$. Analogously to $(2.34)$, we may write that (see $[10$, p. 63$]$ )

$$
\left\|f_{1}\right\|_{\mathcal{A}(\mathbb{I})}:=\sum_{m=0}^{\infty}\left|\hat{f}_{1}(m)\right|=\sum_{m=0}^{\infty}|\hat{f}(m, 0)|
$$

and

$$
\left\|f_{2}\right\|_{\mathcal{A}(\mathbb{I})}:=\sum_{n=0}^{\infty}\left|\hat{f}_{2}(n)\right|=\sum_{n=0}^{\infty}|\hat{f}(0, n)| .
$$


Combining these with (2.34) gives

$$
\|f\|_{\mathcal{A}\left(\mathbb{I}^{2}\right)}=\sum_{m=1}^{\infty} \sum_{n=1}^{\infty}|\hat{f}(m, n)|+\left\|f_{1}\right\|_{\mathcal{A}(\mathbb{I})}+\left\|f_{2}\right\|_{\mathcal{A}(\mathbb{I})}-|\hat{f}(0,0)| .
$$

Assume $\left\|f_{1}\right\|_{\mathcal{A}(\mathbb{I})}<\infty$ and $\left\|f_{2}\right\|_{\mathcal{A}(\mathbb{I})}<\infty$, then the double Walsh-Fourier series in (2.6) is absolutely convergent if and only if

$$
\sum_{m=1}^{\infty} \sum_{n=1}^{\infty}|\hat{f}(m, n)|<\infty
$$

Thus, in order to obtain sufficient conditions for the absolute convergence of the double Walsh-Fourier series in (2.6), we have to combine the sufficient conditions for the absolute convergence of single Walsh-Fourier series (see [10, pp. 64-68]) with Theorems 2.1-2.3 and Corollaries 2.1-2.4 in the previous section. As an illustration, we present two simple corollaries.

We recall that the dyadic analogue of Bernstein's famous theorem for trigonometric Fourier series (see [10, Corollary 3 on p. 65]) says that if a function $g: \mathbb{I} \rightarrow \mathbb{R}$ is such that $g \in \operatorname{Lip}(\alpha ; W)$ for some $\alpha>1 / 2$, then $g \in \mathcal{A}(\mathbb{I})$. Combining this theorem with our Corollary 2.1 for $p=2$ and $r=1$ yields the following

Corollary 2.5. If a function $f: \mathbb{I}^{2} \rightarrow \mathbb{R}$ is such that $f \in \operatorname{Lip}\left(\alpha_{1}, \alpha_{2} ; W\right)$, $f_{1} \in \operatorname{Lip}\left(\alpha_{3} ; W\right)$ and $f_{2} \in \operatorname{Lip}\left(\alpha_{4} ; W\right)$ for some $\alpha_{j}>1 / 2, j=1,2,3,4$; where $f_{1}$ and $f_{2}$ are defined in (2.36) and (2.37), then $f \in \mathcal{A}\left(\mathbb{I}^{2}\right)$.

We recall that the dyadic analogue of Zygmund's famous theorem for single trigonometric series (see [10, Corollary 4 on p. 67]) says that if a function $g: \mathbb{I} \rightarrow \mathbb{R}$ is such that $g \in \operatorname{Lip}(\alpha ; W) \cap B F_{s}(\mathbb{I})$ for some $\alpha>0$ and $0<s<2$, then $g \in \mathcal{A}(\mathbb{I})$. Combining this theorem with our Corollary 2.3 for $r=1$ yields the following 
Corollary 2.6. If a funtion $f: \mathbb{I}^{2} \rightarrow \mathbb{R}$ is such that $f \in \operatorname{Lip}\left(\alpha_{1}, \alpha_{2} ; W\right) \cap$ $B F_{s}\left(\mathbb{I}^{2}\right), f_{1} \in \operatorname{Lip}\left(\alpha_{3} ; W\right) \cap B F_{s_{1}}(\mathbb{I})$ and $f_{2} \in \operatorname{Lip}\left(\alpha_{4} ; W\right) \cap B F_{s_{2}}(\mathbb{I})$ for some $\alpha_{j}>0, j=1,2,3,4$ and $0<s, s_{1}, s_{2}<2$, then $f \in \mathcal{A}\left(\mathbb{I}^{2}\right)$. 


\section{Summary}

Our thesis is based on the classical results of Bernstein and Zygmund giving sufficient conditions for the absolute convergence of the Fourier series of a complex valued function with period $2 \pi$. Namely, if the function $f(x)$ satisfies the Lipschitz condition of order $\alpha$ where $\alpha>1 / 2$, or is of bounded variation and satisfies the Lipshitz condition of order $\alpha$ where $\alpha>0$, then its Fourier series converges absolutely.

Many generalizations of these theorems were proved, for example by Szász [12], Salem [9] and the latest one by Gogoladze and Meskhia [4]. In the first part of our thesis we extend these results from single to double Fourier series. Let $f \in L^{1}\left(\mathbb{T}^{2}\right)$, where $\mathbb{T}^{2}:=[-\pi, \pi) \times[-\pi, \pi)$ is the two dimensional torus. Consider the double series

$$
\sum_{m \in \mathbb{Z}} \sum_{n \in \mathbb{Z}} \gamma_{m n}|\hat{f}(m, n)|^{r}
$$

where $\gamma_{m n}$ is a double sequence, $r \in \mathbb{R}_{+}$and the Fourier coefficients $\hat{f}(m, n)$ are defined by

$$
\hat{f}(m, n):=\frac{1}{4 \pi^{2}} \iint_{\mathbb{T}^{2}} f(x, y) e^{-i(m x+n y)} d x d y, \quad(m, n) \in \mathbb{Z}^{2} .
$$

We give sufficient conditions for the convergence of the series in (1) in terms of moduli of continuity, of bounded $s$-variation in the sense of Vitali or Hardy and Krause.

We give some corollaries as an application of our theorems in some particular case, for example, when $\gamma_{m n} \equiv 1$ or $\gamma_{m n}=m^{\beta_{1}} n^{\beta_{2}}$ and $r=1$. 
As to the Fourier coefficients $\hat{f}(m, n)$ in the special case when $n=0$ and $m=0$, we proceed as follows

(2) $\quad \hat{f}(m, 0)=\hat{f}_{1}(m), \quad$ where $\quad f_{1}(x):=\frac{1}{2 \pi} \int_{\mathbb{T}} f(x, y) d y, x \in \mathbb{T}$

and

$$
\hat{f}(0, n)=\hat{f}_{2}(n), \quad \text { where } \quad f_{2}(x):=\frac{1}{2 \pi} \int_{\mathbb{T}} f(x, y) d x, y \in \mathbb{T} .
$$

In the special case when $\gamma_{m n} \equiv 1$ and $r=1$ the conditions of our theorems ensure the absolute convergence of the double Fourier series of $f$, hence the extension of the theorems of Bernstein and Zygmund reads as follows.

If a function $f: \mathbb{T}^{2} \rightarrow \mathbb{R}$ is such that $f \in \operatorname{Lip}\left(\alpha_{1}, \alpha_{2}\right), f_{1} \in \operatorname{Lip}\left(\alpha_{3}\right)$ and $f_{2} \in \operatorname{Lip}\left(\alpha_{4}\right)$ for some $\alpha_{j}>1 / 2, j=1,2,3,4$; where $f_{1}$ and $f_{2}$ are defined in (2) and (3), then the double Fourier-series of $f$ converges absolutely.

If a function $f: \mathbb{T}^{2} \rightarrow \mathbb{R}$ is such that $f \in \operatorname{Lip}\left(\alpha_{1}, \alpha_{2}\right) \cap B V_{H}\left(\overline{\mathbb{T}}^{2}\right), f_{1} \in$ $\operatorname{Lip}\left(\alpha_{3}\right) \cap B V(\overline{\mathbb{T}})$ and $f_{2} \in \operatorname{Lip}\left(\alpha_{4}\right) \cap B V(\overline{\mathbb{T}})$ for some $\alpha_{j}>0, j=1,2,3,4$ and $0<s, s_{1}, s_{2}<2$; where $f_{1}$ and $f_{2}$ are defined in (2) and (3), then the double Fourier-series of $f$ converges absolutely.

In the last section of the first part we extend our results to multiple Fourier series.

The second part of our thesis is about the Walsh system and the absolute convergence of double Walsh-Fourier series. Let $f \in L^{1}\left(\mathbb{I}^{2}\right)$, where $\mathbb{I}^{2}:=$ $[0,1) \times[0,1)$. Consider the double series

$$
\sum_{m=0}^{\infty} \sum_{n=0}^{\infty} \gamma_{m n}|\hat{f}(m, n)|^{r}
$$

where $\gamma_{m n}$ is a double sequence, $r \in \mathbb{R}_{+}$and the Walsh-Fourier coefficients $\hat{f}(m, n)$ are defined by

$$
\hat{f}(m, n):=\int_{0}^{1} \int_{0}^{1} f(x, y) w_{m}(x) w_{n}(x) d x d y, \quad(m, n) \in \mathbb{N}^{2},
$$


where $w_{m}(x)$ is the $m$ th Walsh-function. Refer to the work of F. Móricz [5] on the absolute convergence of single Walsh-Fourier series, we give sufficient conditions for the convergence of the series in (4) in terms of (either global or local) dyadic moduli of continuity and bounded $s$-fluctuation of $f$.

According to (2) and (3), in the special case when $n=0$ and $m=0$, we proceed as follows

$$
\hat{f}(m, 0)=\hat{f}_{1}(m), \quad \text { where } \quad f_{1}(x):=\int_{0}^{1} f(x, y) d y, \quad x \in \mathbb{I}
$$

and

$$
\hat{f}(0, n)=\hat{f}_{2}(n), \quad \text { where } \quad f_{2}(y):=\int_{0}^{1} f(x, y) d x, \quad y \in \mathbb{I} .
$$

In the special case when $\gamma_{m n} \equiv 1$ and $r=1$ the conditions of our theorems ensure the absolute convergence of the double Walsh-Fourier series of $f$, hence the extension of the dyadic analogue of the theorems of Bernstein and Zygmund reads as follows.

If a function $f: \mathbb{I}^{2} \rightarrow \mathbb{R}$ is such that $f \in \operatorname{Lip}\left(\alpha_{1}, \alpha_{2} ; W\right), f_{1} \in \operatorname{Lip}\left(\alpha_{3} ; W\right)$ and $f_{2} \in \operatorname{Lip}\left(\alpha_{4} ; W\right)$ for some $\alpha_{j}>1 / 2, j=1,2,3,4$; where $f_{1}$ and $f_{2}$ are defined in (2.36) and (2.37), then the Walsh-Fourier series of $f$ converges absolutely.

If a funtion $f: \mathbb{I}^{2} \rightarrow \mathbb{R}$ is such that $f \in \operatorname{Lip}\left(\alpha_{1}, \alpha_{2} ; W\right) \cap B F_{s}\left(\mathbb{I}^{2}\right), f_{1} \in$ $\operatorname{Lip}\left(\alpha_{3} ; W\right) \cap B F_{s_{1}}(\mathbb{I})$ and $f_{2} \in \operatorname{Lip}\left(\alpha_{4} ; W\right) \cap B F_{s_{2}}(\mathbb{I})$ for some $\alpha_{j}>0, j=$ $1,2,3,4$ and $0<s, s_{1}, s_{2}<2$, then the Walsh-Fourier series of $f$ converges absolutely. 


\section{Bibliography}

[1] E. Berkson and T. A. Gillespie, Absolutely continuous functions of two variables and well-bounded operators J. London Math. Soc (2), 30 (1984), 305-321

[2] S. Bernstein, Sur la convergence absolue des séries trigonométriques, C.R. Acad. Sci. Paris Sér. I. Math., 158 (1914), 1661-1664.

[3] J.A. Clarkson and C.R. Adams, On definitions of bounded variation for functions of two variables, Trans. Amer. Math. Soc., 35 (1933), 824-854.

[4] L. Gogoladze and R. Meskhia, On the absolute convergence of trigonometric Fourier series Proc. Razmadze Math. Inst. 141 (2006), 29-40

[5] F. Móricz, Absolute convergence of Walsh-Fourier series and related results Analysis Math. 36 (2010), 275-286

[6] F. Móricz and A. Veres, Absolute convergence of multiple Fourier series Acta Math. Hungar. 117 (2007), 275-292

[7] F. Móricz and A. Veres, Absolute convergence of multiple Fourier series revisited Analysis Math. 34 (2008), 145-162

[8] F. Móricz and A. Veres, Absolute convergence of double Walsh-Fourier series and related results Acta Math. Hungar. (to appear)

[9] R. Salem, On a theorem of Zygmund, Duke Math. J., 10 (1943), 23-31. 
[10] F. Schipp, W.R. Wade, P. Simon and J. Pl, Walsh Series: an Introduction to Dyadic Harmonic Analysis, Adam Hilger, Bristol and New York, 1990.

[11] E.M. Stein and G. Weiss, Introduction to Fourier Analyis on Euclidean Spaces, Princeton Univ. Press, 1971.

[12] Szász, Über der Konvergenzexponenten der Fourierschen Reihen gewisser Funktionenklassen, Sitzungsberichte der Akademie München 1922, 135-150.

[13] P.L. Ul'yanov, Series with respect to a Haar system with monotone coefficients (in Russian), Izv. Akad. Nauk SSSR Ser. Mat., 28 (1964), 925-950.

[14] A. Veres, Extensions of the theorems os Szász and Zygmund on the absolute convergence of Fourier series Acta Sci. Math. 74 (2008), 191206

[15] Z. Waraszkiewicz, Remarque sur un théorème de M. Zygmund, Bull. Internat. Acad. Polonaise, 1929, 275-279.

[16] N. Wiener, The quadratic variation of a function and its Fourier coefficients, Massachusetts J. Math., 3 (1924), 72-94.

[17] A. Zygmund, Remarque sur la convergence absolue des séries de Fourier, J. London Math. Soc., 3 (1928), 194-195.

[18] A. Zygmund, Some points in the theory of trigonometric and power series, Trans. Amer. Math. Soc., 36 (1934), 586-617.

[19] A. Zygmund, Trigonometric series, Cambridge Univ. Press (Cambridge, U.K., 1959). 